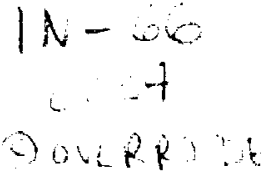

\title{
Model Reduction of Nonsquare Linear MIMO Systems Using Multipoint Matrix Continued-fraction Expansions $\dagger$
}

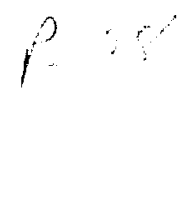

hy' TONG-YI GUO

Department of Chemical Engineering. National Kaohsiung Institute of Technology, Kaohsiung. Taiwan 807, Republic of China

CHYI HWANG $\ddagger$

Department of Chemical Engineering. National Chung Cheng University, Chia-Yi, Taiwan 621, Republic of China

and LEANG-SAN SHIFH

Department of Electrical Engineering, University of Houston, Houston, TX 77204-4793, U.S.A.

ABSTRACT: This paper deals with the multipoint Caucr matrix continued-fraction expansion (MCFE) for mode'/ reduction of line'ar multi-input multi-output (MIMO) systems with tarious numbers of inputs and outputs. A salient feature of the proposed MCFE approach to model reduction of MIMO systems with square transfer matrices is its equiralence to the matrix Pade approximation approach. The' Cauer second form of the ordinary MCFE for a square transfer function matrix is generalized in this paper to a multipoint and nonsquare-matrix cersion. An interesting connection of the multipoint Cauce MCFE mothod to the multipoint matrix Pade approximation method is established. Also, algorithms for obtaining the reduceddearec matrix-fraction descriptions and reduced-dimensional state-space models from a transfer function matrix bia the multipoint Cater MCFE algorithm are presented. Practical adeantages of using the multipoint Cater MCFE are discussed and anmerical example is provided to illustrate the algorithms.

\section{Introduction}

The accurate mathematical modeling or accurate identification of linear timeinvariant multi-input multi-output (MIMO) systems usually leads to high-degree

+This work was supported by the National Science Council of the Republic of China under Grant NSC82-0402-E006-247, the U.S. Army Research Office under Contract DAAL-03-87-K0001 and the NASA-Johnson Space Center under Grants NAG-9-380 and NAG-9-385.

† Author to whom all correspondence should be addressed. 
transfer-function matrices or high-dimensional state-space models. The analysis and design of such high-degree transfer-function matrices in the frequency domain, or such high-dimensional state-space models in the time domain, is not an easy task because of computational difficulties and implementation considerations. There is thus a real incentive to reduce the complexity of linear time-invariant MIMO models to equivalent simple ones.

Several methods are available in the literature (1-6) for approximating a linear time-invariant MIMO system by a reduced-order model. Among them, the method of matrix continued fraction expansion (2-12) has the distinct advantages of computational simplicity and applicability to obtain both frequency-domain as well as time-domain reduced-order models. Chen (2) used a matrix Cauer second form of continued fractions to obtain a reduced-order model by retaining the first several significant matrix partial quotients and discarding the others. Owing to its equivalence to the matrix Pade approximation about $s=0$, the method of using the Cauer second form of MCFE provides satisfactory results in the steady-state response only.

To remove this drawback, Shieh and Gaudiano $(9,10)$ have proposed an MCFE involving expansion points at $s=0$ and $s=\infty$, which is equivalent to the matrix Padé approximation about $s=0$ and $s=\infty$. More recently, Chen and Hwang (7) have presented a multipoint MCFE method to produce better reduced-order models for linear time-invariant MIMO systems.

It is worth noting that the mentioned MCFE methods are derived for the MIMO systems having an equal number of inputs and outputs while being described by their matrix-fraction description (MFD). For broadening the application scope of the MCFE method to include the nonsquare MIMO systems, Shieh et al. (11, 13) have proposed the use of a matrix pseudo-inverse for the Cauer second form of the MCFE. Their method is only applicable to an MIMO system described by its MFD and the connection of the MCFE method to the matrix Pade approximation method has not been explored yet.

The main purpose of this paper is two-fold. Firstly we wish to generalize the CFE algorithm of multipoint Padé fitting (14) to the matrix version for obtaining the reduced-degree MFDs and reduced-dimensional state-space models of a nonsquare linear MIMO system characterized by its transfer-function matrix or its MFD. Secondly we want to reveal the connection of the multipoint Cauer MCFE method to the multipoint matrix Pade approximation method.

The paper is organized as follows. In Section II, computational algorithms involving a matrix pseudo-inverse are derived for determining the multipoint Cauer MCFE of a nonsquare linear time-invariant MIMO system from its transferfunction matrix and MFD, respectively. In Section III, an efficient recursive algorithm is presented for obtaining a set of MFDs with different reduced degrees from the multipoint Cauer MCFE algorithm. Also, the connection of the multipoint Cauer MCFE method involving the matrix pseudo-inverse to the multipoint matrix Padé approximation method is established. In Section IV, a canonical block staterealization of an MFD based on its multipoint Cauer MCFE representation is presented for obtaining reduced-dimensional state-space models. An explicit matrix-relationship between the multipoint Cauer MCFE canonical form and the 
block companion form is also derived. Such a matrix-relationship is useful in connection with the construction of an aggregation matrix which relates the state vectors of the system and its reduced-dimensional models.

Throughout this paper, the following notations are used:

$\mathbf{A}^{*}$ : left pseudo-inverse of the matrix $\mathbf{A}$, defined as $\left(\mathbf{A}^{\mathrm{T}} \mathbf{A}\right)^{\prime} \mathbf{A}^{\mathrm{T}}$;

$\mathbf{A}^{-}$: right pscudo-inverse of the matrix $\mathbf{A}$, defined as $\mathbf{A}^{1}\left(\mathbf{A} \mathbf{A}^{\mathrm{T}}\right)^{\prime}$;

$\mathbf{I}_{n}$ : identity matrix of order $n$;

$\mathbf{O}_{n}$ : null matrix of order $n$;

$\mathbf{O}_{p \times 4}: p$ by $q$ null matrix:

$\mathbf{R}^{\prime \prime 4}$ : set of $p \times q$ real matrices.

\section{Multipoint Cauer MCFE of a Nonsquare Transfer-function Matrix}

A linear time-invariant $q$-input $p$-output system may be described by the $p \times q$ transfer-function matrix

$$
\mathbf{G}(s)=\left[g_{i, j}(s)\right], \quad i=1,2 \ldots, p ; \quad j=1,2, \ldots, q
$$

or by the matrix-fraction description (MFD) (15):

$$
\mathbf{G}(\mathbf{s})=\left[\sum_{i=0}^{N} \mathbf{A}_{2, i} s^{i}\right]\left[\sum_{i=0}^{N} \mathbf{A}_{1, i} s^{i}\right]^{\prime} \triangleq \mathbf{A}_{2}(s) \mathbf{A}_{1}(s) \quad, \quad p \geqslant q
$$

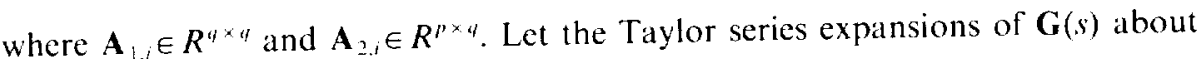
the $m$ distinct real points $\sigma_{1}, \sigma_{2}, \ldots, \sigma_{m}$, be

$$
\mathbf{G}(s)=\sum_{j=0}^{s} \mathbf{T}_{i, j}\left(s-\sigma_{i}\right)^{i}, \quad i=1,2, \ldots, m
$$

where

$$
\mathbf{T}_{i, i}=\left.{ }_{j ! \mathrm{d} s^{\prime}}^{1} \mathrm{~d}^{\prime} \mathbf{G}(s)\right|_{s=\sigma_{i}} \in R^{p \times 4} .
$$

In the following, we deal with the MCFE for the nonsquare transfer-function matrix $\mathbf{G}(s)$. Before proceeding, let $\mu_{1}, \mu_{2}, \ldots, \mu_{m}$ be the multiplicities corresponding to the distinct real points $\sigma_{1}, \sigma_{2}, \ldots, \sigma_{m}$, such that $\mu_{i}+\mu_{2}+\cdots+\mu_{m}=2 N$. These numbers $\sigma_{i}$ will be used as expansion points taking into account multiplicities. Let

$$
\left(s_{1}, s_{2}, \ldots, s_{2 N}\right)=(\underbrace{\sigma_{1}, \ldots, \sigma_{1}}_{\mu_{1}}, \underbrace{\sigma_{2}, \ldots, \sigma_{2}, \ldots, \sigma_{m}, \ldots, \sigma_{m}}_{\mu})
$$

denote the ordered point system. Also, let

$$
v_{i}=\mu_{1}+\mu_{2}+\cdots+\mu_{i} \quad \text {. }
$$

\subsection{Multipoint Cauer MCFE of an MFD}

From the definition of a matrix pseudo-inverse and the MFD (2), we can write $\mathbf{G}(s)$ as

$$
\mathbf{G}(s)=\left[\mathbf{G}^{\#}(s)\right]^{\sim}=\left[\left[\mathbf{A}_{2}(s) \mathbf{A}_{1}{ }^{1}(s)\right]^{\#}\right]^{\sim}=\left[\mathbf{A}_{1}(s) \mathbf{A}_{2}^{\#}(s)\right]^{\sim} .
$$

Yol 3318. No. 2, pp. 189-216. 1994 
Tong-Yi Guo et al.

Assume that $\mathbf{A}_{1}^{\#}\left(s_{1}\right)$ exists and let

$$
\mathbf{H}_{1}=\mathbf{A}_{1}\left(s_{1}\right) \mathbf{A}_{2}^{*}\left(s_{1}\right) \in R^{4 \times p} .
$$

Then, we can expand $\mathbf{A}_{1}(s) \mathbf{A}_{2}^{*}(s)$ as

$$
\mathbf{A}_{1}(s) \mathbf{A}_{2}^{\#}(s)=\mathbf{H}_{1}+\left(s-s_{1}\right) \mathbf{A}_{3}(s) \mathbf{A}_{2}^{\#}(s)
$$

where the matrix polynomial

$$
\mathbf{A}_{3}(s)=\sum_{j=0}^{N-1} \mathbf{A}_{3, j} s^{j}, \quad \mathbf{A}_{3, j} \in R^{4 \times 4}
$$

is to be determined. Postmultiplying both sides of (7) by $\mathbf{A}_{2}(s)$ and then using the identity $\mathbf{A}_{2}^{\#}(s) \mathbf{A}_{2}(s)=\mathbf{I}_{4}$ yields the relation:

$$
\mathbf{A}_{1}(s)=\mathbf{H}_{1} \mathbf{A}_{2}(s)+\left(s-s_{1}\right) \mathbf{A}_{3}(s) .
$$

Substituting the matrix polynomials of $\mathbf{A}_{1}(s), \mathbf{A}_{2}(s)$ and $\mathbf{A}_{3}(s)$ into (9), and then equating the coefficients of like powers, we obtain

$$
\mathbf{A}_{3, j}=\mathbf{A}_{1, j+1}-\mathbf{H}_{1} \mathbf{A}_{2, j+1}+s_{1} \mathbf{A}_{3, j+1}, \quad j=N-1, N-2, \ldots, 0
$$

where $\mathbf{A}_{2, N}=\mathbf{O}_{p \times q}$ and $\mathbf{A}_{3, N}=\mathbf{O}_{q}$.

With the substitution of (7) into (5), G(s) can be further written as

$$
\mathbf{G}(s)=\left[\mathbf{H}_{1}+\left(s-s_{1}\right) \mathbf{A}_{3}(s) \mathbf{A}_{2}^{*}(s)\right]^{-}=\left[\mathbf{H}_{1}+\left(s-s_{1}\right)\left[\mathbf{A}_{2}(s) \mathbf{A}_{3}{ }^{\prime}(s)\right]^{*}\right]^{\sim} .
$$

Similarly, assume that $\mathbf{A}_{3}^{-1}\left(s_{2}\right)$ exists, and let

$$
\mathbf{H}_{2}=\mathbf{A}_{2}\left(s_{2}\right) \mathbf{A}_{3}{ }^{\prime}\left(s_{2}\right) \in R^{p \times q}
$$

then the term in the inner brackets of (11) can be expanded as

$$
\mathbf{A}_{2}(s) \mathbf{A}_{3}^{-1}(s)=\mathbf{H}_{2}+\left(s-s_{2}\right) \mathbf{A}_{4}(s) \mathbf{A}_{3}{ }^{\prime}(s)
$$

where the matrix polynomial

$$
\mathbf{A}_{4}(s)=\sum_{j=0}^{N} \mathbf{A}_{4, j} s^{j}, \quad \mathbf{A}_{4, j} \in R^{p \times 4}
$$

satisfies the relation:

$$
\mathbf{A}_{2}(s)=\mathbf{H}_{2} \mathbf{A}_{3}(s)+\left(s-s_{2}\right) \mathbf{A}_{4}(s) .
$$

From this we have

$$
\mathbf{A}_{4, j}=\mathbf{A}_{2, j+1}-\mathbf{H}_{2} \mathbf{A}_{3, j+1}+s_{2} \mathbf{A}_{4, j+1}, \quad j=N-2, N-1, \ldots, 0
$$
where $\mathbf{A}_{4, j}=\mathbf{O}_{n \times 4}$ for $j \geqslant N-1$. With the substitution of (13) into (11), $\mathbf{G ( s )}$ is
now expanded as

$$
\mathbf{G}(s)=\left[\mathbf{H}_{1}+\left(s-s_{1}\right)\left[\mathbf{H}_{2}+\left(s-s_{2}\right) \mathbf{A}_{4}(s) \mathbf{A}_{3}^{\prime}(s)\right]^{*}\right]^{-} .
$$

By repeating the expansion procedure of (5)-(17) in the MFD, $\mathbf{A}_{4}(s) \mathbf{A}_{3}{ }^{\prime}(s)$, we can finally expand $\mathbf{G}(s)$ into the following multipoint Cauer matrix continued 
fractions:

$$
\begin{aligned}
\mathbf{G}(s)=\left[\mathbf{H}_{1}+\left(s-s_{1}\right)\left[\mathbf{H}_{2}+\left(s-s_{2}\right)\left[\mathbf{H}_{3}+\left(s-s_{3}\right)\right.\right.\right. & {\left.\left.\left.\left[\begin{array}{ll}
\mathbf{H}_{2 N} & 1 \\
& \left.+\left(\begin{array}{lll}
s-s_{2 N} & 1
\end{array}\right) \mathbf{H}_{2 N}^{*}\right]^{\sim} \ldots
\end{array}\right]^{\#}\right]^{\sim}\right]^{\#}\right] . }
\end{aligned}
$$

The matrix partial quotients $\mathbf{H}_{i}$ can be obtained via the recurrence relations:

$$
\begin{aligned}
\mathbf{H}_{i}= & \begin{cases}\mathbf{A}_{i}\left(s_{i}\right) \mathbf{A}_{i+1}^{*}\left(s_{i}\right), & i=1,3, \ldots, 2 N-1 \\
\mathbf{A}_{i}\left(s_{i}\right) \mathbf{A}_{i+1}\left(s_{i}\right), & i=2,4, \ldots, 2 N\end{cases} \\
\mathbf{A}_{i+2}(s)= & \sum_{i=1)}^{N} \mathbf{A}_{i+2, j} s^{j} \\
= & 1 \\
s-s_{i} & \left(\mathbf{A}_{i}(s)-\mathbf{H}_{i} \mathbf{A}_{i+1}(s)\right) \\
\mathbf{A}_{i+2, j} & =\mathbf{A}_{i, j+1}-\mathbf{H}_{i} \mathbf{A}_{i+1, j+1}+s_{i} \mathbf{A}_{i+2, j+1}, \\
& j=N-i_{2}-1, N-i_{2}-2, \ldots, 0
\end{aligned}
$$

where $i_{2}$ denotes the integer part of $i / 2$ and the required matrix inverse and matrix pseudo-inverse are assumed to exist. For convenience, we can construct an expansion array as shown in Table I for evaluating $\mathbf{A}_{i, j}$. In this array, the first two matrix rows are taken from the matrix polynomials $\mathbf{A}_{1}(s)$ and $\mathbf{A}_{2}(s)$, respectively. The subsequent matrix rows are evaluated using $(18 \mathrm{a}-\mathrm{c})$.

\subsection{Multipoint Cater MCFE hased on Taylor-series expansions}

In this subsection, we derive an alternative tabular algorithm for obtaining the matrix partial quotients $\mathbf{H}_{i}$ of the multipoint Cauer MCFE (18) of the matrix transfer function $\mathbf{G}(s)$ from its Taylor-series coefficients $\mathbf{T}_{i,}$ in (3) for $j=0,1, \ldots, \mu_{i}-1$ and $i=1, \ldots, m$.

To begin with, we define

$$
\begin{aligned}
\mathbf{G}_{i, j}(s) & =\mathbf{G}_{i, j, 1)}+\mathbf{G}_{i, j, 1}\left(s-\sigma_{i}\right)+\mathbf{G}_{i, j, 2}\left(s-\sigma_{i}\right)^{2}+\cdots \\
& \triangleq \sum_{k=0}^{\prime} \mathbf{G}_{i, j, k}\left(s-\sigma_{i}\right)^{k}
\end{aligned}
$$

TABLE I

Expansion array for evaluating $\mathbf{A}_{\mathrm{i}, \mathrm{j}}$

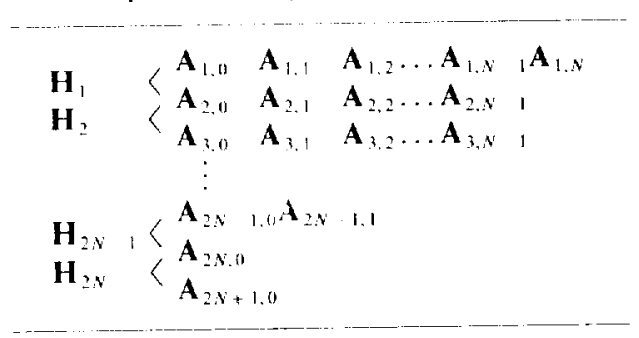


with

$$
\begin{aligned}
\mathbf{G}_{i, 1}(s) & =\mathbf{I}_{q}+\mathbf{O}_{i}\left(s-\sigma_{i}\right)+\mathbf{O}_{4}\left(s-\sigma_{i}\right)^{2}+\cdots \\
& \triangleq \sum_{k=0}^{\ell} \mathbf{G}_{i, 1, k}\left(s-\sigma_{i}\right)^{k}, \quad i=1,2, \ldots, m
\end{aligned}
$$

and

$$
\begin{aligned}
\mathbf{G}_{i, 2}(s) & =\mathbf{T}_{i, 0}+\mathbf{T}_{i, 1}\left(s-\sigma_{i}\right)+\mathbf{T}_{i, 2}\left(s-\sigma_{i}\right)^{2}+\cdots \\
& \triangleq \sum_{k=0}^{\infty} \mathbf{G}_{i, 2 . k}\left(s-\sigma_{i}\right)^{k}, \quad i=1,2, \ldots, m .
\end{aligned}
$$

Then, from (3a), (20) and (21), we can represent $\mathbf{G}(s)$ by one of the following infinite-term MFDs:

$$
\begin{aligned}
\mathbf{G}(s) & =\mathbf{G}_{i, 2}(s) \mathbf{G}_{i, 1}{ }^{\prime}(s) \\
& =\left[\mathbf{G}_{i, 1}(s) \mathbf{G}_{i, 2}^{\#}(s)\right]^{-}, \quad i=1,2, \ldots, m .
\end{aligned}
$$

According to the form of the multipoint Cauer MCFE (18) and the expansion ordered point system (4), we know that matrix partial quotients $\mathbf{H}_{v_{i}+1}, \mathbf{H}_{\mathbf{v}_{i}+2}, \ldots$, $\mathbf{H}_{v_{i}+\mu_{i}}$ are evaluated from the infinite-term $\operatorname{MFD~}_{i, 2}(s) \mathbf{G}_{i, 1}^{-1}(s)$. Since the multipoint Cauer MCFE involves $m$ distinct expansion points $\sigma_{i}, i=1,2, \ldots, m$, it is therefore required to manipulate the $m$ infinite-term MFDs in (22), which represent the same transfer-function matrix $\mathbf{G}(s)$, to obtain the partial quotients $\mathbf{H}_{i}$ of the multipoint Cauer MCFE (18).

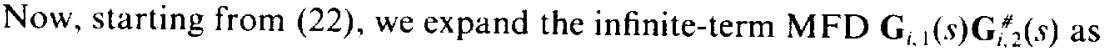

$$
\mathbf{G}_{i, 1}(s) \mathbf{G}_{i, 2}^{*}(s)=\mathbf{H}_{1}+\left(s-s_{1}\right) \mathbf{G}_{i, 3}(s) \mathbf{G}_{i, 2}^{\#}(s), \quad i=1,2, \ldots, m
$$

where

$$
\begin{aligned}
\mathbf{H}_{1} & =\mathbf{G}_{1,1}\left(s_{1}\right) \mathbf{G}_{1,2}^{\#}\left(s_{1}\right) \text { for } s_{1}=\sigma_{1} \\
& =\mathbf{G}_{1,1,0} \mathbf{G}_{1,2,0}^{\#} .
\end{aligned}
$$

In $(23)$, each of the infinite-term series $\mathbf{G}_{i, 3}(s)$ satisfies

$$
\mathbf{G}_{i, 1}(s)=\mathbf{H}_{1} \mathbf{G}_{i, 2}(s)+\left(s-s_{1}\right) \mathbf{G}_{i, 3}(s), \quad i=1,2, \ldots, m
$$

which is obtained by postmultiplying both sides of $(23)$ with $\mathbf{G}_{i, 2}(s)$ and using the identity $\mathbf{G}_{i, 2}^{\#}(s) \mathbf{G}_{i, 2}(s)=\mathbf{I}_{q}$. Substituting the series representations of $\mathbf{G}_{i, 1}(s), \mathbf{G}_{i, 2}(s)$ and $\boldsymbol{G}_{i, 3}(s)$ into $(25)$, we have

$$
\begin{aligned}
\sum_{k=0}^{x} \mathbf{G}_{i, 1, k}\left(s-\sigma_{i}\right)^{k}=\mathbf{H}_{1} \sum_{k \neq 1}^{\infty} \mathbf{G}_{i, 2, k}\left(s-\sigma_{i}\right)^{k} & \\
& +\left[\left(s-\sigma_{i}\right)+\left(\sigma_{i}-s_{1}\right)\right] \sum_{k=1}^{\infty} \mathbf{G}_{i, 3, k}\left(s-\sigma_{i}\right)^{k} .
\end{aligned}
$$

Equating the coefficients of like terms $\left(s-\sigma_{i}\right)^{k}$, we obtain 


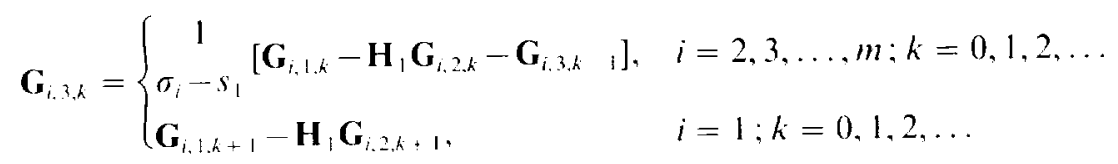

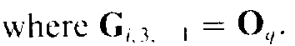

In general, the MFDs, $\mathbf{G}_{i, j}(s) \mathbf{G}_{i, j+1}^{*}(s)$ for $j$ odd and $\mathbf{G}_{i, j}(s) \mathbf{G}_{i, j+1} 1(s)$ for $j$ even, can respectively be expanded as

$$
\mathbf{G}_{i, j}(s) \mathbf{G}_{i, i}^{*}(s)=\mathbf{H},+\left(s-s_{,}\right) \mathbf{G}_{i, j+2}(s) \mathbf{G}_{i, j+1}^{\#}(s), \quad i=1,1+1, \ldots, m
$$

and

$$
\mathbf{G}_{t, j}(s) \mathbf{G}_{i, j+1}^{1}(s)=\mathbf{H}_{i}+\left(s-s_{j}\right) \mathbf{G}_{i, j, 2}(s) \mathbf{G}_{i, j+1}^{1}(s), \quad i=l, l+1, \ldots, m
$$

where $s_{j}=\sigma_{l}$ and

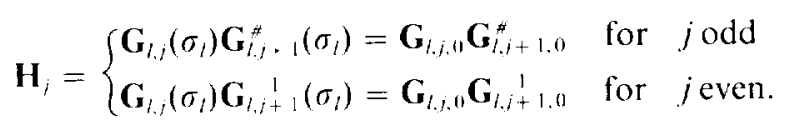

In (28). each infinite-term series $\mathbf{G}_{i, t}$. $2(s)$ satisfies

$$
\mathbf{G}_{i, j}(s)=\mathbf{H}_{i} \mathbf{G}_{i, j, 1}(s)+\left(s-s_{j}\right) \mathbf{G}_{i, j+2}(s), \quad i=1,1+1, \ldots, m
$$

i.e.

$$
\begin{aligned}
\sum_{k}^{\prime} \mathbf{G}_{i, j, k}\left(s-\sigma_{i}\right)^{k}=\mathbf{H}_{i} \sum_{k}^{\prime} \mathbf{G}_{i, j, 1, k}\left(s-\sigma_{i}\right)^{k} & \\
& +\left[\left(s-\sigma_{i}\right)+\left(\sigma_{i}-s_{j}\right)\right] \sum_{k=01}^{\prime} \mathbf{G}_{i, j+2, k}\left(s-\sigma_{i}\right)^{k} .
\end{aligned}
$$

This last equation allows one to evaluate the coefficients $\mathbf{G}_{i, j}, 2, h$ for $i=1,1+1, \ldots, m$ and $k=0,1,2, \ldots$ as follows :

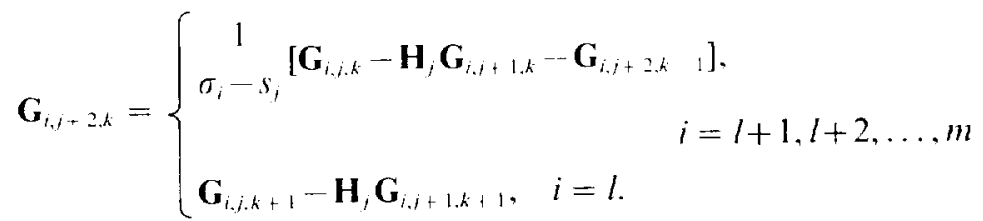

Following the above derivation, we can construct $m$ expansion arrays as shown in Table II for calculating the matrix partial quotients $\mathbf{H}_{1}, \mathbf{H}_{2}, \ldots, \mathbf{H}_{2 N}$ of the multipoint Cauer MCFE of G(s) from the matrix coefficients of the Taylor series expansions in (3).

In the expansion array in Table II, the first two matrix rows are specified by

$$
\begin{aligned}
& \mathbf{G}_{i, 1 . h}= \begin{cases}\mathbf{I}_{i} & \text { for } k=0 \\
\mathbf{O}_{q} & \text { for } k=1,2, \ldots, \mu_{i}-\mathbf{l}\end{cases} \\
& \mathbf{G}_{i, 2, k}=\mathbf{T}_{i, k} \text { for } k=0,1, \ldots, \mu_{i}-1 .
\end{aligned}
$$


TABLF: II

Expansion array for evaluating $\mathbf{G}_{\mathrm{i}, \mathrm{j}, \mathrm{k}}$

\begin{tabular}{|c|c|c|c|c|c|c|}
\hline & $\begin{array}{l}\mathbf{G}_{i, 1,0} \\
\mathbf{G}_{t, 2,0} \\
\mathbf{G}_{i, 3,0}\end{array}$ & $\begin{array}{l}\mathbf{G}_{i, 1,1} \\
\mathbf{G}_{1,2,1} \\
\mathbf{G}_{i, 3,3}\end{array}$ & $\begin{array}{l}\mathbf{G}_{1,1,2} \\
\mathbf{G}_{i, 2,2} \\
\mathbf{G}_{i, 3,2}\end{array}$ & $\begin{array}{l}\cdots \\
\cdots \\
\ldots\end{array}$ & $\begin{array}{l}\mathbf{G}_{i, 1, \mu_{1}}= \\
\mathbf{G}_{i, 2, \mu_{1}}= \\
\mathbf{G}_{i, \mu_{1}}=\end{array}$ & $\begin{array}{l}\mathbf{G}_{t, 1, \mu_{i}} \\
\mathbf{G}_{t, 2, \mu_{1}} \\
\mathbf{G}_{i, 1, \mu_{1}}\end{array}$ \\
\hline $\begin{array}{l}\mathbf{H}_{\mathbf{v}_{1}+1} \\
\mathbf{H}_{\mathbf{v}_{1}+2}\end{array}$ & 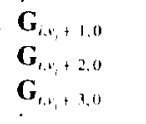 & $\begin{array}{l}\mathbf{G}_{i, w_{1}+1,1} \\
\mathbf{G}_{i, w_{1}+2,1} \\
\mathbf{G}_{1, w_{i}+3.1}\end{array}$ & $\begin{array}{l}\mathbf{G}_{i,,+1,2} \\
\mathbf{G}_{i, v_{1}, 2,2} \\
\mathbf{G}_{i, v_{1}+3,2}\end{array}$ & $\begin{array}{l}\cdots \\
\cdots \\
\cdots\end{array}$ & 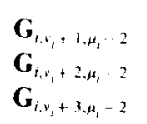 & $\begin{array}{l}\mathbf{G}_{t, b_{t}+1, t_{t}} \\
\mathbf{G}_{i, x_{1}+2, \mu_{t}}\end{array}$ \\
\hline $\begin{array}{l}\mathbf{H}_{v_{2}+\mu_{i} \cdot 1} \\
\mathbf{H}_{v_{r}+\mu_{i}}\end{array}$ & 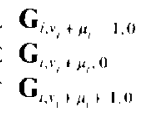 & $\begin{array}{l}\mathbf{G}_{i, w_{i}+n_{i}-1,1} \\
\mathbf{G}_{i, w_{i}+\mu_{i}, 1}\end{array}$ & $\boldsymbol{G}_{t, x_{1}+\mu_{1}} \quad 1.2$ & & & \\
\hline
\end{tabular}

The subsequent matrix rows of the expansion array are obtained by the following recurrence formulas,

$$
\mathbf{G}_{i, j+2, k}=\left\{\begin{aligned}
1 & {\left[\mathbf{G}_{i, k, k}-\mathbf{H}_{j} \mathbf{G}_{i, j+1, k}-\mathbf{G}_{i, j+2, k} \quad 1\right], } \\
\sigma_{i}-s_{j} & =0,1, \ldots, \mu_{i}-1 \text { and } j=1,2, \ldots, v_{i} \\
\mathbf{G}_{i, j, k+1} & -\mathbf{H}_{i} \mathbf{G}_{i, j+1, k+1} \\
k & =0,1, \ldots, v_{i}+\mu_{i}-j-1 \text { and } j=v_{i}+1, v_{i}+2, \ldots, v_{i}+\mu_{i}-1
\end{aligned}\right.
$$

which are derived from the relation in (30). The matrix partial quotients $\mathbf{H}_{i}$ for $j=v_{i}+1, v_{i}+2, \ldots, v_{i}+\mu_{i}$ associated with the expansion array in Table II are evaluated as

$$
\mathbf{H}_{j}= \begin{cases}\mathbf{G}_{i, j, 0} \mathbf{G}_{i, j+1,0}^{*} & \text { for } j \text { odd } \\ \mathbf{G}_{i, j, 0} \mathbf{G}_{i, j+1,0} 1 & \text { for } j \text { even }\end{cases}
$$

It is noted that in the foregoing derivations, we have assumed that the required matrix inverse and matrix pseudo-inverse exist. If $\mathbf{G}_{i, j, 0}^{\prime}$ or $\mathbf{G}_{i, j, 0}^{\#}$ for $i=1,2, \ldots, m$ do not exist, we should readjust the expansion points $\sigma_{i}$ such that the matrix inverse exists. When all coefficients of any matrix row in the expansion array are null, the expansion procedure terminates. In this case, we can use the calculated matrix partial quotients and the inversion procedure in the next section to derive the MFD for $\mathbf{G}(s)$.

\section{Reduced-degree MFDs Derived from the Multipoint Cauer MCFE}

Suppose the transfer-function matrix $\mathbf{G}(s)$ of a high-degree $q$-input $p$-output system is expanded into a multipoint Cauer MCFE in (18). An $M$ th-degree reduced $\mathrm{MFD}, \hat{\mathrm{G}}_{M}(s)$, for the system can be obtained by truncating the matrix partial 
quotients $\mathbf{H}_{2 M+1}, \mathbf{H}_{2 M+2}, \ldots, \mathbf{H}_{2 N}$ in (18). Hence $\hat{\mathbf{G}}_{M}(s)$ is given by

$$
\begin{aligned}
\hat{\mathbf{G}}_{M}(s)=\left[\mathbf{H}_{1}+\left(s-s_{1}\right)\left[\mathbf{H}_{2}+\left(s-s_{2}\right)\left[\mathbf{H}_{3}\right.\right.\right. & +\left(s-s_{3}\right)\left[\ldots \left[\mathbf{H}_{2 M}\right.\right. \\
& \left.\left.\left.\left.+\left(s-s_{2 M}, \mathbf{l}_{2 M}\right) \mathbf{H}_{2 M}^{*} \ldots\right]^{*}\right]^{-}\right]^{*}\right]^{-} .
\end{aligned}
$$

To convert the truncated Cauer MCFE into its MFD, we define

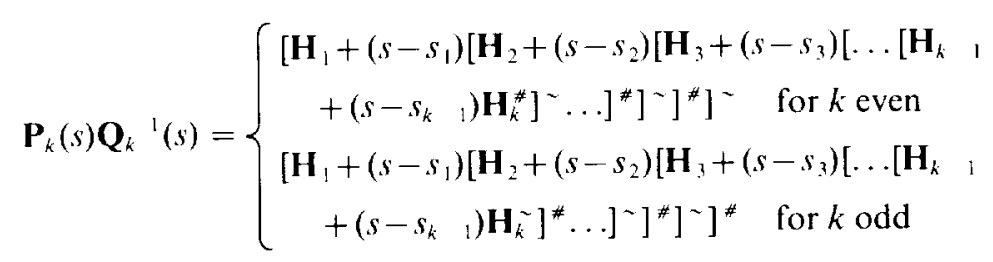

where

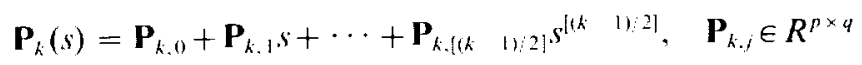

$$
\begin{aligned}
& \mathbf{Q}_{k}(s)=\mathbf{Q}_{k, 0}+\mathbf{Q}_{k, 1} s+\cdots+\mathbf{Q}_{k,\left[k: 2 ! s^{[k, 2]},\right.} \mathbf{Q}_{k, i} \in R^{4 \times 4}
\end{aligned}
$$

and $[r]$ denotes the integer part of $r$. Then it can be shown that the matrix polynomials $\mathbf{P}_{k}(s)$ and $\mathbf{Q}_{k}(s)$ satisfy the following recurrence relations:

$$
\begin{aligned}
& \mathbf{P}_{k}(s)=\mathbf{P}_{k} \quad,(s) \mathbf{H}_{k}+\left(s-s_{k} \quad 1\right) \mathbf{P}_{k} \quad 2(s), \quad k=1,2, \ldots \\
& \mathbf{Q}_{k}(s)=\mathbf{Q}_{k} \quad{ }_{1}(s) \mathbf{H}_{k}+\left(s-s_{k} \quad{ }_{1}\right) \mathbf{Q}_{k} \quad{ }_{2}(s), \quad k=1,2, \ldots
\end{aligned}
$$

where $\mathbf{P}_{01}(s)=\mathbf{O}_{p \times 4}, \mathbf{P}_{1}(s)=\mathbf{O}_{p}, \mathbf{Q}_{0}(s)=\mathbf{I}_{q}$, and $\mathbf{Q}_{1}(s)=\mathbf{H}_{1}$. Using the relations in (38). we can construct an inversion array as shown in Table III to compute the matrix coefficients $\mathbf{P}_{k, i}$ and $\mathbf{Q}_{k, i}$ from the matrix partial quotients $\mathbf{H}_{j}$.

In the inversion array, the first two matrix rows are given by

$$
\mathbf{P}_{0,0}=\mathbf{O}_{p \times q}, \quad \mathbf{P}_{1.0}=\mathbf{I}_{p}, \quad \mathbf{Q}_{0,0}=\mathbf{I}_{q,}, \quad \mathbf{Q}_{1.0}=\mathbf{H}_{1}
$$

TABLE [I]

Inersion array

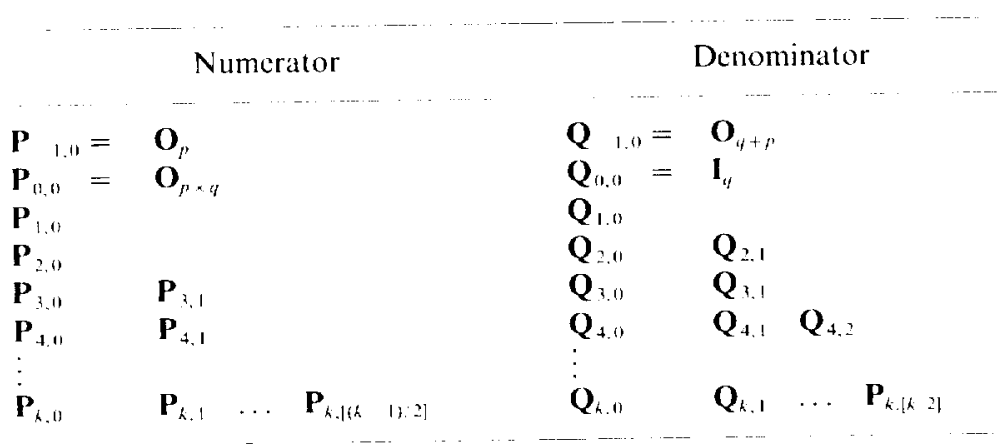

Vol. 3318, No. 2, pp. 189216,1994 
Tong- Yi Guo et al.

and the remaining matrix entries are evaluated using the following recurrence relations :

$$
\begin{aligned}
& \mathbf{P}_{k, j}=\mathbf{P}_{k, 1, j} \mathbf{H}_{k}-s_{k} \quad{ }_{1} \mathbf{P}_{k} \quad{ }_{2, j}+\mathbf{P}_{k} \quad 2 . j, i, j=0,1, \ldots,[(k-1) / 2] \\
& \mathbf{Q}_{k, j}=\mathbf{Q}_{k} \quad{ }_{1, j} \mathbf{H}_{k}-s_{k} \quad \mathbf{l}_{k} \mathbf{Q}_{2, j}+\mathbf{Q}_{k} \quad{ }_{2, j} \quad, \quad j=0,1, \ldots,[k / 2]
\end{aligned}
$$

where

$$
\mathbf{P}_{k .1}= \begin{cases}\mathbf{O}_{p \times 4} & \text { for } k \text { even } \\ \mathbf{O}_{p} & \text { for } k \text { odd }\end{cases}
$$

and

$$
\mathbf{Q}_{k . \quad 1}= \begin{cases}\mathbf{Q}_{q} & \text { for } k \text { even } \\ \mathbf{O}_{a \times p} & \text { for } k \text { odd }\end{cases}
$$

for $k=1,2, \ldots, M$.

Once the inversion array is developed, the $M$ th-degree reduced MFD, which corresponds to the truncated MCFE in (35), can be readily written as

$$
\begin{aligned}
\hat{\mathbf{G}}_{M}(s)= & \mathbf{P}_{2 M}(s) \mathbf{Q}_{2 M}(s) \\
= & {\left[\mathbf{P}_{2 M, 0}+\mathbf{P}_{2 M, 1} s+\cdots+\mathbf{P}_{2 M M-1} s^{M}{ }^{\prime}\right]\left[\mathbf{Q}_{2 M .0}\right.} \\
& \left.+\mathbf{Q}_{2 M, 1} s+\cdots+\mathbf{Q}_{2 M, M} s^{M}\right] \text {. }
\end{aligned}
$$

It is important here to examine the link between the $M$ th-degree reduced MFD, $\hat{\mathbf{G}}_{M}(s)$ and the original transfer-function matrix $\mathbf{G}(s)$. As a specific example, we consider $\hat{\mathbf{G}}_{1}(s)$ which has the following explicit form:

$$
\hat{\mathbf{G}}_{1}(s)=\mathbf{H}_{2}\left[\mathbf{H}_{1} \mathbf{H}_{2}+\left(s-s_{1}\right) \mathbf{I}_{q}\right] \text { ' }
$$

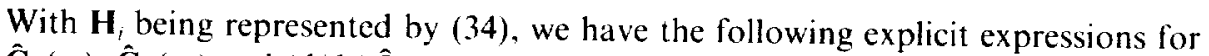
$\hat{\mathbf{G}}_{1}\left(s_{1}\right), \hat{\mathbf{G}}_{1}\left(s_{2}\right)$ and $(\mathrm{d} / \mathrm{d} s) \hat{\mathbf{G}}_{1}\left(s_{1}\right)$ :

$$
\begin{aligned}
& \hat{\mathbf{G}}_{1}\left(s_{1}\right)=\mathbf{H}_{2}\left[\mathbf{H}_{1} \mathbf{H}_{2}\right] \\
& = \begin{cases}\mathbf{G}_{1,2,0} \mathbf{G}_{1,3,0}^{1}\left[\mathbf{G}_{1,1,0} \mathbf{G}_{1,2,0}^{*} \mathbf{G}_{1,2,0} \mathbf{G}_{1,3,0}\right] & \text { for } s_{2}=s_{1} \\
\mathbf{G}_{2,2,0} \mathbf{G}_{2,3,0}\left[\mathbf{G}_{1,1,0} \mathbf{G}_{1,2,0}^{*} \mathbf{G}_{2,2,0} \mathbf{G}_{2,3,0}\right] & \text { for } s_{2} \neq s_{1}\end{cases} \\
& \hat{\mathbf{G}}_{1}\left(s_{2}\right)=\mathbf{H}_{2}\left[\mathbf{H}_{1} \mathbf{H}_{2}+\left(s_{2}-s_{1}\right) \mathbf{I}_{4}\right] \\
& =\mathbf{G}_{2,2,0}\left[\mathbf{H}_{1} \mathbf{G}_{2,2,0}-\left(s_{2}-s_{1}\right) \mathbf{G}_{2,3,0}\right] \quad \text { । } \\
& =\mathbf{G}_{2,2,0} \mathbf{G}_{2,1,0}{ }^{1}=\mathbf{G}_{2,2,0} \\
& \mathrm{~d} \hat{\boldsymbol{G}}_{1}\left(s_{1}\right)=-\mathbf{H}_{2}\left[\left(\boldsymbol{H}_{1} \mathbf{H}_{2}\right)^{2}\right] \quad \text { । } \\
& = \begin{cases}-\mathbf{G}_{1,2,0} \mathbf{G}_{1,3,0}=\mathbf{G}_{1,2,0} \mathbf{G}_{1,2,0}^{\#} \mathbf{G}_{1,2,1} & \text { for } s_{2}=s_{1} \\
-\mathbf{G}_{2,2,0} \mathbf{G}_{2,3,0}=\left[\left(\mathbf{G}_{1,2,0}^{\#} \mathbf{G}_{2,2,0}\right)^{2}\right] & \text { for } s_{2} \neq s_{1} .\end{cases}
\end{aligned}
$$


Comparing (43) and (21), we have

$$
\begin{array}{ll}
\hat{\mathbf{G}}_{1}\left(s_{1}\right)=\mathbf{G}\left(s_{1}\right), & \hat{\mathbf{G}}_{1}\left(s_{2}\right)=\mathbf{G}\left(s_{2}\right) \text { for } s_{1} \neq s_{2} \text { and } p=q \\
\hat{\mathbf{G}}_{1}\left(s_{1}\right)=\mathbf{G}\left(s_{1}\right), & \mathrm{d} \hat{\mathbf{G}}_{1}\left(s_{2}\right)=\frac{\mathrm{d}}{\mathrm{d} s} \mathbf{G}\left(s_{2}\right) \text { for } s_{1}=s_{2} \text { and } p=q \\
\hat{\mathbf{G}}_{1}\left(s_{1}\right) \neq \mathbf{G}\left(s_{1}\right), & \hat{\mathbf{G}}_{1}\left(s_{2}\right)=\mathbf{G}\left(s_{2}\right) \text { for } p \neq q .
\end{array}
$$

By a similar direct deduction, we can obtain the link between the $M$ th-degree $(M<N)$ reduced MFD, $\hat{\mathbf{G}}_{M}($.$) , in (41) and the original transfer-function matrix$ $\mathbf{G}(s)$ as follows:

(i) $\operatorname{squate} \operatorname{system}(p=q)$

$$
\left.\mathrm{d}^{\mathrm{d}^{\prime}} \hat{\mathbf{G}}_{M}(s)\right|_{\sigma_{1}}=\left.{ }_{\mathrm{d} s^{\prime}}^{\mathrm{d}^{\prime}} \mathbf{G}(s)\right|_{s, \sigma_{i}} \text { for } j=0,1, \ldots, \hat{\mu}_{i}-1 \text { and } i=1,2, \ldots l
$$

where $/$ is the number of distinct points that appear in the ordered point system, and

$$
\left(s_{1}, s_{2}, \ldots, s_{2 w}\right)=\left(\sigma_{1}, \ldots, \sigma_{1}, \sigma_{2}, \ldots, \sigma_{2}, \ldots, \sigma_{1}, \ldots, \sigma_{t}, \sigma_{1}, \ldots, \sigma_{l}\right)
$$

which is obtained by truncating the last $2 N-2 M$ points of the ordered point system in (4):

(ii) nonsquare $\operatorname{system}(p \neq q)$

$$
\left.\stackrel{\mathrm{d}^{\prime}}{\mathrm{d} s^{\prime}} \hat{\mathbf{G}}_{M}(s)\right|_{,-\sigma_{i}}=\left.{ }_{\mathrm{d} s^{\prime}}^{\mathrm{d}^{j}} \mathbf{G}(s)\right|_{s=\sigma} \text { for } \bar{\mu}_{j}>0, j=0,1, \ldots, \bar{\mu}_{i}-1 \text { and } 1 \leqslant i \leqslant l
$$

where $\bar{\mu}_{i}$, the multiplicity of the point $\sigma_{i}$, appears in the ordered point system.

$$
\left(s_{2}, s_{4}, \ldots, s_{2 M}\right)=\left(\sigma_{1}, \ldots, \sigma_{1}, \sigma_{2}, \ldots, \sigma_{2}, \ldots, \sigma_{1}, \ldots, \sigma_{l}\right.
$$

which is obtained from $(45)$ by deleting the odd-indexed elements $s_{2 i}$ for $j=1,2, \ldots, M$.

Notice from (46) that for a nonsquare system, the $M$ th-degree reduced MFD obtained by the multipoint Cauer MCFE has the same Pade properties only around the even expansion points. However, since the multipoint Cauer MCFE involves the matrix pseudo-inverse in the calculation of odd matrix partial quotients, the obtained reduced-degree MFDs approximate the Pade properties around the odd 
Tong-Yi Guo et al.

expansion points, i.e.

$$
\left.\left.\frac{\mathrm{d}^{j}}{\mathrm{~d} s^{j}} \hat{\mathbf{G}}_{M}(s)\right|_{s=\sigma_{i}} \approx \mathrm{d}_{\mathrm{d} s^{j}}^{\mathrm{d}^{j}} \mathbf{G}(s)\right|_{s-\sigma,} \text { for } j=0,1, \ldots, \hat{\mu}_{i}-\bar{\mu}_{i} \text { and } 1 \leqslant i \leqslant l
$$

where the expansion point $\sigma_{i}$ appears $\hat{\mu}_{i}-\bar{\mu}_{i}(>0)$ times in the ordered point system, and

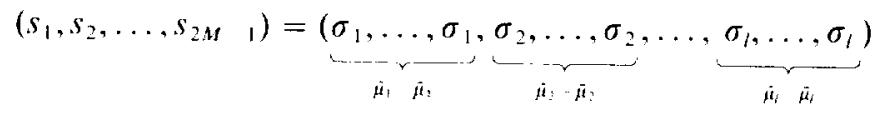

which is obtained from (43) by dropping the even-indexed elements $s_{2}$ for $j=1,2, \ldots, M$.

\section{State-space Models Obtained from the Multipoint Cauer MCFE}

4.1. State-space formulation of the multipoint Cauer MCFE

Let $\mathbf{Y}(s)$ and $\mathbf{U}(s)$ be, respectively, the Laplace transforms of the output and input vectors of the system. Then $\mathbf{Y}(s)$ is related to $\mathbf{U}(s)$ by

$$
\mathbf{Y}(s)=\mathbf{G}(s) \mathbf{U}(s) .
$$

Premultiplying both sides of (48) by $\mathbf{G}^{\#}(s)$ and using (18), we have

$$
\begin{aligned}
& \mathbf{U}(s)=\mathbf{G}^{\#}(s) \mathbf{Y}(s) \\
& =\left[\mathbf{H}_{1}+\left(s-s_{1}\right)\left[\mathbf{H}_{2}+\left(s-s_{2}\right) \mathbf{G}_{3}(s)\right]^{\sharp}\right] \mathbf{Y}(s) \\
& =\mathbf{H}_{1} \mathbf{Y}(s)+\left[\begin{array}{c}
1 \\
\left(s-s_{1}\right)
\end{array}\left(\mathbf{H}_{2}+r_{1} \mathbf{G}_{3}(s)\right)+\mathbf{G}_{3}(s)\right]^{*} \mathbf{Y}(s)
\end{aligned}
$$

where

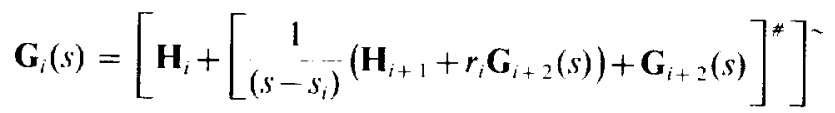

and $r_{i}=s_{i}-s_{i+1}$ for $i=1,3,5, \ldots, 2 N-1$. Let

$$
\mathbf{X}_{1}(s)=\left[\begin{array}{c}
1 \\
s-s_{1}
\end{array}\left(\mathbf{H}_{2}+r_{1} \mathbf{G}_{3}(s)\right)+\mathbf{G}_{3}(s)\right]^{*} \mathbf{Y}(s)
$$

Then, we have

$$
\mathbf{Y}(s)=\left[\begin{array}{c}
1 \\
s-s_{1}
\end{array}\left(\mathbf{H}_{2}+r_{1} \mathbf{G}_{3}(s)\right)+\mathbf{G}_{3}(s)\right] \mathbf{X}_{1}(s)
$$

and

$$
\mathbf{X}_{1}(s)=\mathbf{U}(s)-\mathbf{H}_{1} \mathbf{Y}(s)
$$

The above two relations allow one to construct the block diagram as shown in Fig. 1 for representing the relationship between $\mathbf{Y}(s)$ and $\mathbf{U}(s)$. 


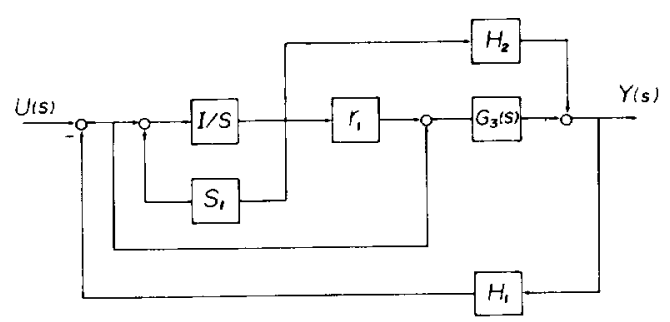

Fici. 1. Block diagram representation of $\mathbf{G}_{1}(s)$.

Using a similar procedure to expand $\mathbf{G}_{3}(s), \mathbf{G}_{5}(s), \ldots$, a realization block diag$\operatorname{ram}($ Fig. 2$)$ for $\mathbf{G}(s)$ can be achieved. It is readily apparent from Fig. 2 that a canonical state-space representation of $\mathbf{G}(s)$ can be written as follows :

$$
\begin{aligned}
\dot{\mathbf{z}} & =\mathbf{H z}+\mathbf{G u} \\
\mathbf{y} & =\mathbf{F} \mathbf{z}
\end{aligned}
$$

with

$$
\begin{aligned}
& \mathbf{z}=\left[\begin{array}{llll}
\mathbf{z}_{1}^{\mathrm{T}} & \mathbf{z}_{2}^{\mathrm{T}} & \ldots & \mathbf{z}_{N}^{\mathrm{T}}
\end{array}\right]^{\mathrm{T}}, \quad \mathbf{z}_{i}=\left[\begin{array}{lllll}
z_{i, 1} & z_{i, 2} & \ldots & z_{i, q}
\end{array}\right]^{\mathrm{T}} \\
& \mathbf{H}=-\mathbf{H}_{0} \mathbf{H}_{\mathrm{c}}+\mathbf{R} \otimes \mathbf{I}_{\mathrm{q}}
\end{aligned}
$$

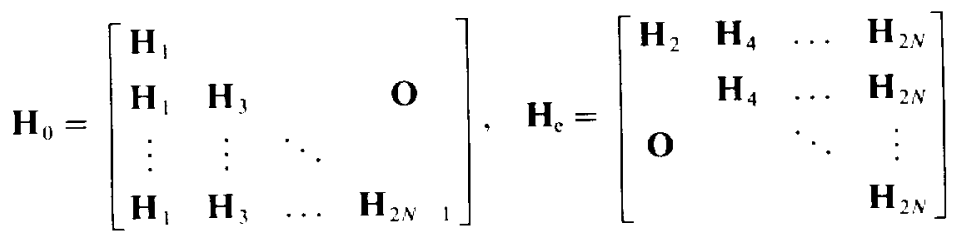

$$
\begin{aligned}
& \mathbf{R}=\left[\begin{array}{ccccccc}
s_{1} & & & & & \\
s_{1} & r_{3} & & & \mathbf{O} & \\
s_{1} & s_{3} & r_{5} & & & \\
\vdots & \vdots & \vdots & \ddots & & & \\
s_{1} & s_{3} & s_{5} & \ldots & r_{2 N} & 3 & \\
s_{1} & s_{3} & s_{5} & \ldots & s_{2 N} & 3 & r_{2 N-1}
\end{array}\right]
\end{aligned}
$$

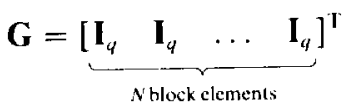

$$
\begin{aligned}
& \mathbf{F}=\left[\begin{array}{llll}
\mathbf{H}_{2} & \mathbf{H}_{4} & \ldots & \mathbf{H}_{2 N}
\end{array}\right]
\end{aligned}
$$

where the superscript $\mathrm{T}$ designates the transpose of a matrix or vector, and $\otimes$ denotes the Kronecker product (16).

\subsection{Link between $M C F E$ and block companion forms}

Let $\mathbf{A}_{1, N}=\mathbf{I}_{4}$, then a controllable block companion form for the realization of the MFD (2) can be written as follows (17): 


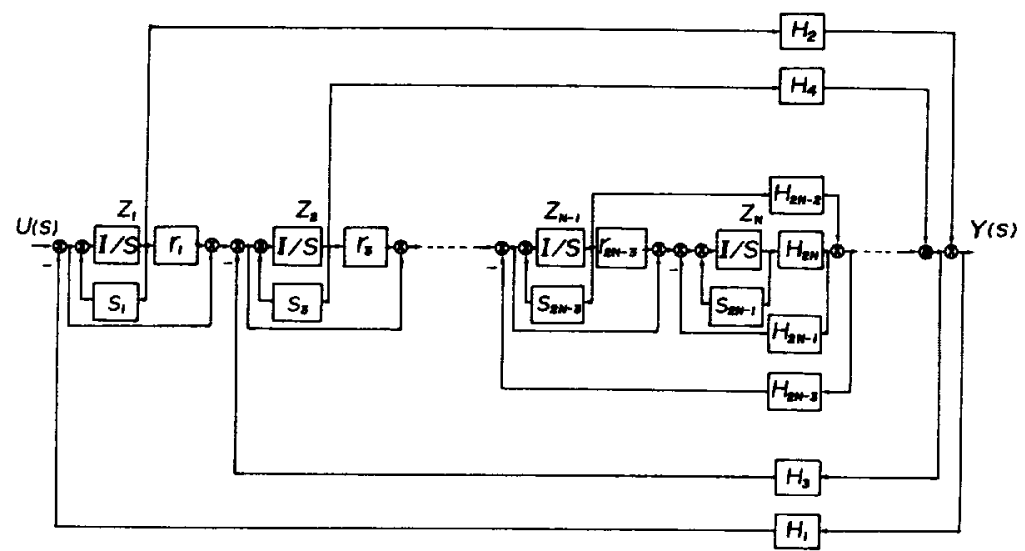

FIG. 2. Realization block diagram of multipoint Cauer matrix CFE.

$$
\begin{aligned}
& \dot{\mathbf{x}}=\mathbf{A x}+\mathbf{B u} \\
& \mathbf{y}=\mathbf{C x}
\end{aligned}
$$

where

$$
\begin{aligned}
\mathbf{x} & =\left[\begin{array}{lllllll}
\mathbf{x}_{1}^{\mathrm{T}} & \mathbf{x}_{2}^{\mathrm{T}} & \ldots & \mathbf{x}_{N}^{\mathrm{T}}
\end{array}\right]^{\mathrm{T}}, \quad \mathbf{x}_{i}=\left[\begin{array}{lllll}
x_{i, 1} & x_{i, 2} & \ldots & x_{i, q}
\end{array}\right]^{\mathrm{T}} \\
\mathbf{A} & =\left[\begin{array}{ccccc}
\mathbf{O}_{q} & \mathbf{I}_{q} & & & \\
\mathbf{O}_{q} & \mathbf{O}_{q} & \mathbf{I}_{q} & & \mathbf{O} \\
\vdots & \vdots & \vdots & \ddots & \\
\mathbf{O}_{q} & \mathbf{O}_{q} & \mathbf{O}_{q} & \ldots & \mathbf{I}_{q} \\
-\mathbf{A}_{1,0} & -\mathbf{A}_{1.1} & -\mathbf{A}_{1,2} & \ldots & -\mathbf{A}_{1, N-1}
\end{array}\right] \\
\mathbf{B} & =\left[\begin{array}{llll}
\mathbf{O}_{4} & \mathbf{O}_{q} & \ldots & \mathbf{I}_{q}
\end{array}\right]^{\mathrm{T}} \\
\mathbf{C} & =\left[\begin{array}{lllll}
\mathbf{A}_{2,0} & \mathbf{A}_{2,1} & \ldots & \mathbf{A}_{2, N} & \ldots
\end{array}\right] .
\end{aligned}
$$

Assume that the system is completely controllable with controllability index equal to $\mathrm{Nq}$. Then there exists a nonsingular block transformation matrix $\mathbf{P}$ which links the block state vectors $\mathbf{z}$ and $\mathbf{x}$ by

$$
\mathbf{z}=\mathbf{P x}
$$

and satisfies the following relations:

$$
\begin{aligned}
\mathbf{P A} & =\mathbf{H P} \\
\mathbf{P B} & =\mathbf{G} \\
\mathbf{C} & =\mathbf{F P} .
\end{aligned}
$$

The desired block similarity transformation matrix $\mathbf{P}$ can be derived from the relation (18a) and (55c). Let the block similarity transformation matrix $\mathbf{P}$ be of 
the form

$$
\mathbf{P}=\left[\begin{array}{cccc}
\mathbf{P}_{1.1} & \mathbf{P}_{1,2} & \ldots & \mathbf{P}_{1, N} \\
\mathbf{P}_{2,1} & \mathbf{P}_{2,2} & \ldots & \mathbf{P}_{2, N} \\
\vdots & \vdots & \ddots & \vdots \\
\mathbf{P}_{N, 1} & \mathbf{P}_{N, 2} & \ldots & \mathbf{P}_{N, N}
\end{array}\right]
$$

where the block elements $\mathbf{P}_{i, j}$ are $q \times q$ matrices. Define the matrix polynomials

$$
\mathbf{P}_{i}(s)=\sum_{j=1}^{N} \mathbf{P}_{i, j} s^{j} \quad, \quad i=1,2, \ldots, N .
$$

Then the relation $(55 \mathrm{c})$ is equivalent to

$$
\mathbf{A}_{2}(s)=\mathbf{H}_{2} \mathbf{P}_{1}(s)+\mathbf{H}_{4} \mathbf{P}_{2}(s)+\cdots+\mathbf{H}_{2 N} \mathbf{P}_{N}(s) .
$$

Rewrite the recursion in (18b) as

$$
\mathbf{A}_{i}(s)=\left(s-s_{i}\right) \mathbf{A}_{i+2}(s)+\mathbf{H}_{i} \mathbf{A}_{i+1}(s), \quad i=1,2, \ldots, 2 N .
$$

Then, by applying the above relations recursively for $i=2,4, \ldots, 2 N$ and noting that $\mathbf{A}_{2 N+2}(s)=\mathbf{O}_{p \times 4}$, we have

$$
\begin{aligned}
\mathbf{A}_{2}(s)=\mathbf{H}_{2} \mathbf{A}_{3}(s)+\left(s-s_{2}\right) \mathbf{H}_{4} \mathbf{A}_{5}(s)+\left(s-s_{2}\right)\left(s-s_{4}\right) \mathbf{H}_{6} \mathbf{A}_{7}(s)+\cdots \\
+\left(s-s_{2}\right) \cdots\left(s-s_{2 N}\right) \mathbf{H}_{2 N} \mathbf{A}_{2 N+1}(s) .
\end{aligned}
$$

Comparing (58) and (60), we obtain

$$
\mathbf{P}_{i}(s)=\prod_{j=1}^{i}\left(s-s_{2 j}\right) \mathbf{A}_{2 i+1}(s), \quad i=1,2, \ldots, N .
$$

Using (57) and equating the coefficients of the like powers of $s^{i}$ in (61), and using the relations in (57), we have the explicit form for $\mathbf{P}_{i, j}$ as

$$
\mathbf{P}_{i, j}=\sum_{k=1}^{i} r_{i, k} \mathbf{A}_{2 i+1, j-k+1}, \quad i=1,2, \ldots, n \text { and } j=1,2, \ldots, n
$$

where $\mathbf{A}_{i, j}$ are the coefficients of the matrix polynomials defined in $(18 \mathrm{~b}, \mathrm{c})$, and $r_{i, k}$ are given by

$$
r_{i, k}= \begin{cases}0 & \text { for } i<k \text { or } k=0 \\ 1 & \text { for } i=k \\ r_{i-1, k, 1}-s_{i-2} r_{i-1, k} & \text { for } i>k\end{cases}
$$

\subsection{Reduced-dimensional state-space models}

Truncation of the last $(2 N-2 M)$ matrix partial quotients of the multipoint Cauer MCFE in (18) after the first $2 M$ matrix partial quotients is equivalent to discarding the $N-M$ inner block loops of Fig. 2. Hence, the multipoint Cauer MCFE canonical state-space model corresponding to the $M$ th-degree $\hat{\mathbf{G}}_{M}(s)$ can 
Tong-Yi Guo et al.

be readily obtained as

where

$$
\begin{aligned}
\dot{\hat{\mathbf{z}}} & =\hat{\mathbf{H}} \hat{\mathbf{z}}+\hat{\mathbf{G}} \mathbf{u} \\
\hat{\mathbf{y}} & =\hat{\mathbf{F}} \hat{\mathbf{z}}
\end{aligned}
$$

$$
\begin{aligned}
& \hat{\mathbf{z}}=\left[\begin{array}{llll}
\hat{\mathbf{z}}_{1}^{\mathrm{T}} & \mathbf{z}_{2}^{\mathrm{T}} & \ldots & \hat{\mathbf{z}}_{M}^{\mathrm{T}}
\end{array}\right]^{\mathrm{T}}, \quad \hat{\mathbf{z}}_{i}=\left[\begin{array}{llll}
\hat{z}_{i, 1} & \hat{z}_{i, 2} & \ldots & \hat{z}_{i, q}
\end{array}\right]^{\mathrm{T}} \\
& \hat{\mathbf{H}}=-\hat{\mathbf{H}}_{0} \hat{\mathbf{H}}_{\mathrm{e}}+\hat{\mathbf{R}} \otimes \mathbf{I}_{q} \\
& \hat{\mathbf{H}}_{0}=\left[\begin{array}{cccc}
\mathbf{H}_{1} & & & \\
\mathbf{H}_{1} & \mathbf{H}_{3} & & \mathbf{O} \\
\vdots & \vdots & \ddots & \\
\mathbf{H}_{1} & \mathbf{H}_{3} & \ldots & \mathbf{H}_{2 M-1}
\end{array}\right], \quad \hat{\mathbf{H}}_{\mathbf{e}}=\left[\begin{array}{cccc}
\mathbf{H}_{2} & \mathbf{H}_{4} & \ldots & \mathbf{H}_{2 M} \\
& \mathbf{H}_{4} & \ldots & \mathbf{H}_{2 M} \\
\mathbf{O} & & \ddots & \vdots \\
& & & \mathbf{H}_{2 M}
\end{array}\right]
\end{aligned}
$$$$
\hat{\mathbf{R}}=\left[\begin{array}{cccccc}
s_{1} & & & & & \\
s_{1} & r_{3} & & & \text { O } & \\
s_{1} & s_{3} & r_{5} & & & \\
\vdots & \vdots & \vdots & \ddots & & \\
s_{1} & s_{3} & s_{5} & \ldots & r_{2 M-3} & \\
s_{1} & s_{3} & s_{5} & \ldots & s_{2 M-3} & r_{2 M-1}
\end{array}\right]
$$

$$
\begin{aligned}
& \hat{\mathbf{G}}=\underbrace{\left[\begin{array}{llll}
\mathbf{I}_{q} & \mathbf{I}_{q} & \ldots & \mathbf{I}_{q}
\end{array}\right.}_{\text {whlock elements }}]^{\mathrm{T}} \\
& \hat{\mathbf{F}}=\left[\begin{array}{llll}
\mathbf{H}_{2} & \mathbf{H}_{4} & \ldots & \mathbf{H}_{2 M}
\end{array}\right] .
\end{aligned}
$$

It is obvious that $\hat{\mathbf{H}}$ is the upper-left $M$ by $M$ block submatrix of $\mathbf{H}$, and $\hat{\mathbf{G}}$ and $\hat{\mathbf{F}}$ are the upper block subvectors of $\mathbf{G}$ and $\mathbf{F}$, respectively. Hence, the truncation procedure in the time domain can be represented by the following mathematical relations :

where

$$
\begin{aligned}
\hat{\mathbf{H}} & =\mathbf{J} \mathbf{H} \mathbf{J}^{\mathrm{T}} \\
\hat{\mathbf{G}} & =\mathbf{J G} \\
\hat{\mathbf{F}} & =\mathbf{F} \mathbf{J}^{\mathrm{T}}
\end{aligned}
$$

$$
\mathbf{J}=\left[\mathbf{I}_{M \varphi}: \mathbf{O}_{M q \times(N \cdots M) q}\right]
$$

Based on the multi-feedback multi-feedforward control theory $(2,10)$ and the realization block diagram of Fig. 2, we know that the inner block states have fewer contributions to the system output. Hence, it is reasonable to consider that for an appropriate $M$, the following approximation holds

$$
\hat{\mathbf{z}} \approx \mathbf{J z} \text {. }
$$

With this approximation and the relation (64), we conclude that the state vector $\hat{\mathbf{z}}$ of the reduced-dimensional state-space model is approximately related to that of the original system in (53) by 


$$
\hat{\mathbf{z}}=\mathbf{Q x}
$$

where

$$
\mathbf{Q}=\mathbf{J P} .
$$

The $\mathbf{Q}$ is called an approximate aggregation matrix. The availability of this approximate aggregation matrix enables the reduced-dimensional state-space models obtained by the multipoint Cauer MCFE method to be useful in designing reduced-order controllers.

\section{An Illustrative Example}

To illustrate the proposed algorithms, we consider a two-input-three-output system characterized by its third-degree MFD

$$
\begin{aligned}
\mathbf{G}(s)= & {\left[\left[\begin{array}{rr}
-7.6051 & -18.4986 \\
-35.0581 & -85.4861 \\
-25.4654 & -62.0624
\end{array}\right]+\left[\begin{array}{rr}
-23.5164 & -49.8596 \\
-4.5654 & 21.8066 \\
-6.4341 & 8.5709
\end{array}\right] s\right.} \\
& \left.+\left[\begin{array}{rr}
-6.6984 & 3.8209 \\
1.8038 & -0.8574 \\
0.2685 & 0.0243
\end{array}\right] s^{2}\right]\left[\begin{array}{rr}
-1.2972 & -2.5216 \\
-85.9888 & -209.6110
\end{array}\right] \\
& +\left[\begin{array}{rr}
-64.1796 & -148.9154 \\
-167.1634 & -326.1264
\end{array}\right] s+\left[\begin{array}{rr}
-16.0738 & 29.8019 \\
-39.1889 & 40.4941
\end{array}\right] s^{2} \\
& \left.+\left[\begin{array}{ll}
1 & 0 \\
0 & 1
\end{array}\right] s^{3}\right] .
\end{aligned}
$$

The transfer-function matrix is given by:

$$
\mathbf{G}(s)=\left[\begin{array}{ll}
g_{1,1}(s) & g_{1,2}(s) \\
g_{2,1}(s) & g_{2,2}(s) \\
g_{3,1}(s) & g_{3,2}(s)
\end{array}\right]
$$

where

$$
\begin{gathered}
g_{1,1}(s)=\left(3.440200+29.863961 s+34.330077 s^{2}-90.583719 s^{3}\right. \\
- \\
g_{2.1}\left(45.025211 s^{4}-6.6984 s^{5}\right) / d(s) \\
g_{3.1}(s)=\left(1.156658+16.036971 s+13.523988 s^{2}-33.627214 s^{3}\right. \\
+
\end{gathered}
$$


Tong-YiGuo et al.

$$
\begin{aligned}
g_{1,2}(s)=\left(4.819364+60.095159 s+200.157141 s^{2}+\right. & 241.049291 s^{3} \\
& \left.+88.349065 s^{4}+3.8209 s^{5}\right) / d(s) \\
g_{2,2}(s)=\left(22.490064+225.973085 s+345.147913 s^{2}+\right. & 23.697755 s^{3} \\
- & \left.18.168391 s^{4}-0.8574 s^{5}\right) / d(s) \\
g_{3,2}(s)=\left(16.293793+163.607382 s+248.927928 s^{2}+\right. & 30.343293 s^{3} \\
& \left.+0.178497 s^{4}+0.0243 s^{5}\right) / d(s) \\
d(s)=55.07803 i+649.225525 s+1817.984559 s^{2} & +1578.243399 s^{3} \\
+ & 126.703614 s^{4}+24.4203 s^{5}+s^{6} .
\end{aligned}
$$

The controllable block companion form corresponding to this MFD is

$$
\begin{aligned}
& {\left[\left[\begin{array}{l}
\dot{x}_{1.1} \\
\dot{x}_{1.2}
\end{array}\right]\right] \quad\left[\begin{array}{ll}
0 & 0 \\
0 & 0
\end{array}\right] \quad\left[\begin{array}{ll}
1 & 0 \\
0 & 1
\end{array}\right]} \\
& {\left[\begin{array}{l}
\dot{x}_{2,1} \\
\dot{x}_{2,2}
\end{array}\right]=\left[\begin{array}{ll}
0 & 0 \\
0 & 0
\end{array}\right] \quad\left[\begin{array}{ll}
0 & 0 \\
0 & 0
\end{array}\right]} \\
& \left.\left[\begin{array}{l}
\dot{x}_{3,1} \\
\dot{x}_{3.2}
\end{array}\right]\right]\left[\begin{array}{rr}
1.2972 & 2.5216 \\
85.9888 & 209.6110
\end{array}\right]\left[\begin{array}{rr}
64.1796 & 148.9154 \\
167.1634 & 326.1264
\end{array}\right] \\
& {\left[\begin{array}{ll}
0 & 0 \\
0 & 0
\end{array}\right]} \\
& {\left[\begin{array}{ll}
1 & 0 \\
0 & 1
\end{array}\right]} \\
& \left.\left.\left.\left[\begin{array}{ll}
16.0738 & -29.8019 \\
39.1889 & -40.4941
\end{array}\right]\right]\left[\begin{array}{l}
x_{3.1} \\
x_{3.2}
\end{array}\right]\right]\left[\begin{array}{ll}
1 & 0 \\
0 & 1
\end{array}\right]\right] \\
& {\left[\begin{array}{l}
y_{1} \\
y_{2} \\
y_{3}
\end{array}\right]=\left[\left[\begin{array}{rr}
-7.6051 & -18.4986 \\
-35.0581 & -85.4861 \\
-25.4654 & -62.0624
\end{array}\right]\left[\begin{array}{rr}
-23.5164 & -49.8596 \\
-4.5654 & 21.8066 \\
-6.4341 & 8.5709
\end{array}\right]\right.} \\
& \left.\left[\begin{array}{rr}
-6.6984 & 3.8209 \\
1.8038 & -0.8574 \\
0.2685 & 0.0243
\end{array}\right]\right]\left[\begin{array}{l}
{\left[\begin{array}{l}
x_{1,1} \\
x_{1,2}
\end{array}\right]} \\
{\left[\begin{array}{l}
x_{2,1} \\
x_{2,2}
\end{array}\right]} \\
{\left[\begin{array}{l}
x_{3,1} \\
x_{3,2}
\end{array}\right]}
\end{array}\right] .
\end{aligned}
$$

The Taylor series expansions of $\mathbf{G}(s)$ around expansion points at $s=0,1,2$ are evaluated as 


$$
\begin{aligned}
& \mathbf{G}(s)=\left[\begin{array}{rr}
0.062460 & 0.087501 \\
-0.041464 & 0.408331 \\
0.021000 & 0.295831
\end{array}\right]+\left[\begin{array}{rr}
-0.194033 & 0.059688 \\
0.040411 & -0.710370 \\
0.043629 & -0.516607
\end{array}\right] s \\
& +\left[\begin{array}{rr}
0.848784 & 0.042327 \\
-0.694785 & 1.161968 \\
-0.961898 & 0.844367
\end{array}\right] s^{2}+\cdots \\
& =\left[\begin{array}{rr}
-0.041074 & 0.140686 \\
-0.041070 & 0.140685 \\
0.000647 & 0.108021
\end{array}\right]+\left[\begin{array}{rr}
-0.091768 & 0.046501 \\
0.012861 & -0.105319 \\
-0.009672 & -0.068097
\end{array}\right](s-1) \\
& +\left[\begin{array}{rr}
0.005283 & -0.005326 \\
0.008434 & 0.058837 \\
0.008503 & 0.042513
\end{array}\right](s-1)^{2}+\cdots \\
& =\left[\begin{array}{rr}
-0.126800 & 0.181971 \\
-0.023260 & 0.071566 \\
-0.003600 & 0.065727
\end{array}\right]+\left[\begin{array}{rr}
-0.078945 & 0.036070 \\
0.020381 & -0.046636 \\
-0.000845 & -0.026606
\end{array}\right](s-2) \\
& +\left[\begin{array}{rr}
0.007431 & -0.005244 \\
0.000944 & 0.014180 \\
0.002074 & 0.009571
\end{array}\right](s-2)^{2}+\cdots
\end{aligned}
$$

Choosing the ordered expansion point system as $\left(s_{1}, s_{1}, s_{2}, s_{2}, s_{3}, s_{3}\right)=(0,0,1,1,2,2)$ and using the recursive algorithms (18) and (33), we construct the expansion arrays as shown in Tables IV and V for evaluating the multipoint Cauer MCFE from the MFD and Taylor series expansion of $\mathbf{G}(s)$, respectively.

We obtain from (18a) and (34), the same set matrix of partial quotients as follows:

$$
\begin{aligned}
& \mathbf{H}_{1}=\left[\begin{array}{rrr}
10.781239 & -5.586419 & 4.521980 \\
0.550334 & 1.447022 & 1.220228
\end{array}\right] \quad \mathbf{H}_{2}=\left[\begin{array}{rr}
0.029679 & 0.095386 \\
-0.019033 & 0.224562 \\
0.010361 & 0.196506
\end{array}\right] \\
& \mathbf{H}_{3}=\left[\begin{array}{rrr}
-10.576133 & -5.571830 & -4.021421 \\
4.017897 & -8.535413 & -4.024899
\end{array}\right] \quad \mathbf{H}_{4}=\left[\begin{array}{rr}
-6.737767 & 3.651197 \\
1.817531 & -1.093386 \\
0.235814 & -0.184328
\end{array}\right] \\
& \mathbf{H}_{5}=\left[\begin{array}{lll}
165.364859 & 70.422714 & 271.800239 \\
209.425455 & 49.768519 & 129.772792
\end{array}\right] \quad \mathbf{H}_{6}=\left[\begin{array}{ll}
0.009688 & 0.074317 \\
0.005302 & 0.011424 \\
0.022325 & 0.012122
\end{array}\right]
\end{aligned}
$$


TABLE IV

Expansion array for evaluating $\mathbf{A}_{\mathrm{i}, \mathrm{j}}$

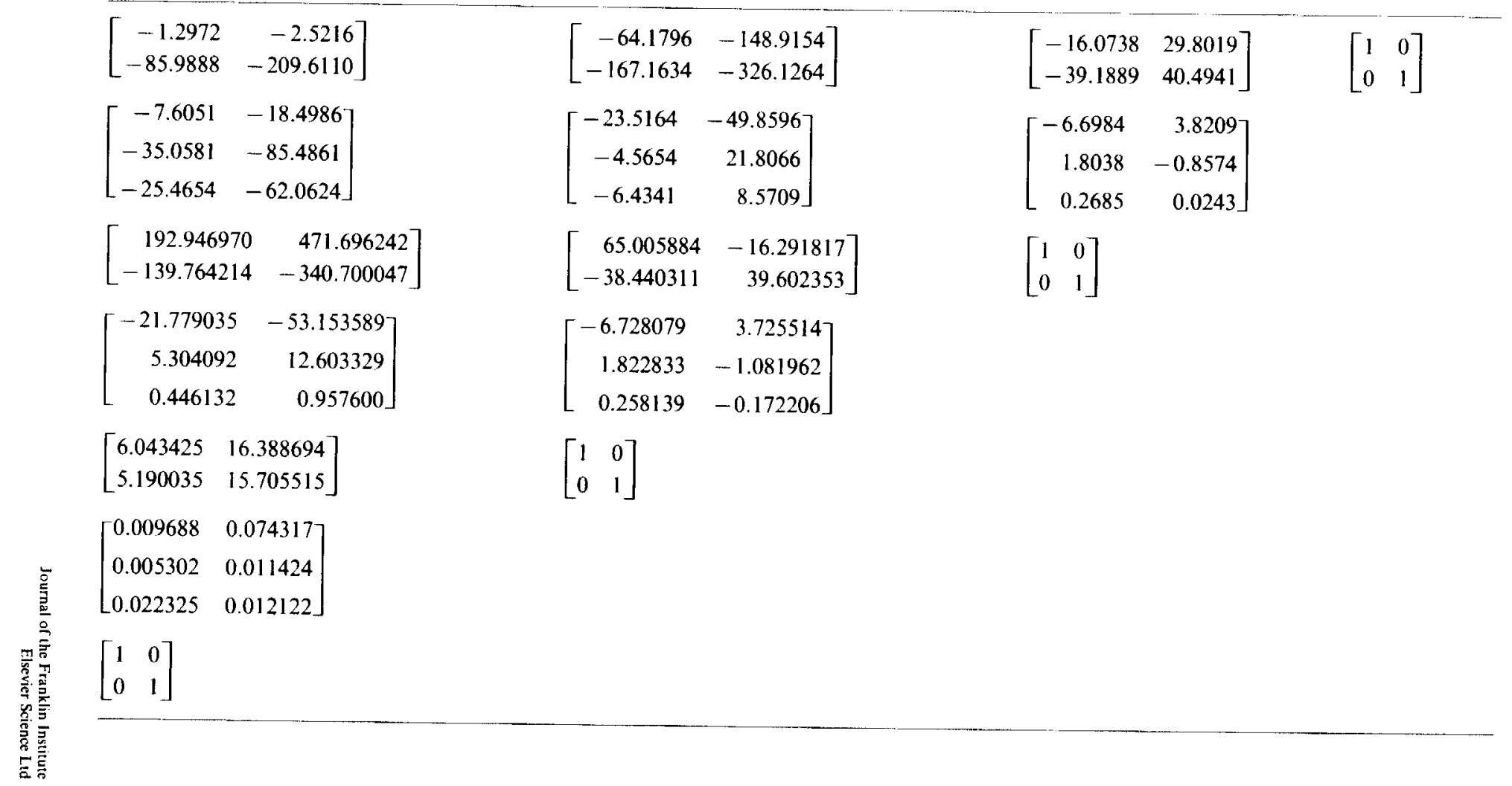


TABle Va

Expansion array for enaluating $\mathbf{G}_{1, \mathrm{j}, \mathrm{k}}$

$\begin{array}{ll}{\left[\begin{array}{ll}1 & 0 \\ 0 & 1\end{array}\right]} & {\left[\begin{array}{ll}0 & 0 \\ 0 & 0\end{array}\right]} \\ {\left[\begin{array}{rr}0.062460 & 0.087501 \\ -0.041464 & 0.408331 \\ 0.021000 & 0.295831\end{array}\right]} & {\left[\begin{array}{rr}-0.194033 & 0.059688 \\ 0.040411 & -0.710370 \\ 0.043629 & -0.516607\end{array}\right]} \\ {\left[\begin{array}{rr}2.120382 & -2.275849 \\ -0.004929 & 1.625451\end{array}\right]} & \end{array}$

TABI.F Vb

Expansion array for evaluating $\mathbf{G}_{2, \mathrm{j} . \mathrm{k}}$

$\begin{array}{ll}{\left[\begin{array}{ll}1 & 0 \\ 0 & 1\end{array}\right]} & {\left[\begin{array}{ll}0 & 0 \\ 0 & 0\end{array}\right]} \\ {\left[\begin{array}{rr}-0.041074 & 0.140686 \\ -0.041070 & 0.140685 \\ 0.000647 & 0.108021\end{array}\right]} & {\left[\begin{array}{rr}-0.091768 & 0.046501 \\ 0.012861 & -0.105319 \\ -0.009672 & -0.068097\end{array}\right]} \\ {\left[\begin{array}{rr}1.210471 & -1.219319 \\ 0.081244 & 0.587192\end{array}\right]} & {\left[\begin{array}{rr}-0.105514 & 0.437559 \\ -0.037548 & -0.377289\end{array}\right]} \\ {\left[\begin{array}{rr}-0.084749 & 0.120865 \\ -0.036275 & -0.014384 \\ -0.027860 & 0.005267\end{array}\right]} & {\left[\begin{array}{rr}-0.000306 & -0.051362 \\ 0.055560 & 0.002118 \\ 0.026659 & -0.003759\end{array}\right]} \\ {\left[\begin{array}{ll}0.308025 & -0.108965 \\ 0.545205 & -0.167977\end{array}\right]} & \end{array}$


Tong-Yi Guo et al.

TABLE Vc

Expansion array for evaluating $\mathbf{G}_{3, \mathrm{j}, \mathrm{k}}$

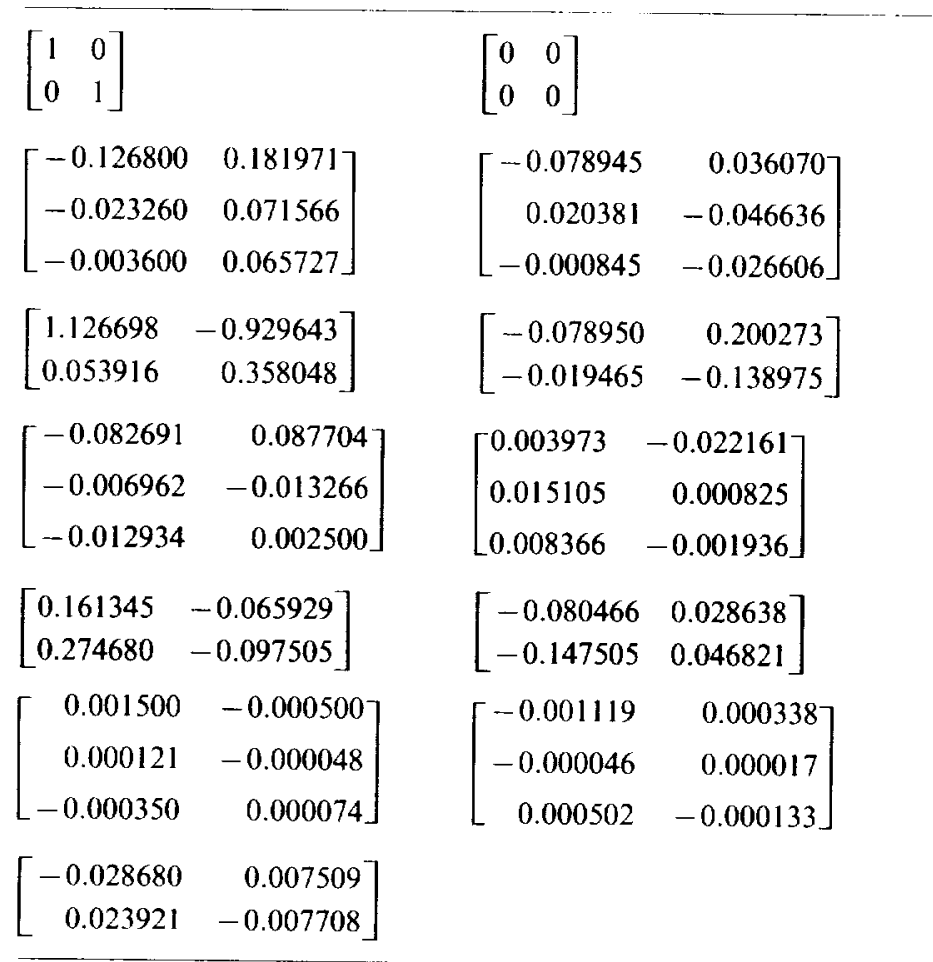

Once the matrix partial quotients $\mathbf{H}_{i}$ are evaluated, the canonical block statespace model corresponding to the multipoint Cauer MCFE can be constructed according to (52) as follows:

$$
\begin{aligned}
& {\left[\begin{array}{l}
{\left[\begin{array}{l}
\dot{z}_{1,1} \\
\dot{z}_{1,2}
\end{array}\right]} \\
{\left[\begin{array}{l}
\dot{z}_{2.1} \\
\dot{z}_{2,2}
\end{array}\right]} \\
{\left[\begin{array}{l}
\dot{z}_{3,1} \\
\dot{z}_{3,2}
\end{array}\right]}
\end{array}\right]=\left[\begin{array}{ll}
{\left[\begin{array}{ll}
-0.473153 & -0.662478 \\
-0.001435 & -0.617223
\end{array}\right]} & {\left[\begin{array}{rr}
81.728620 & -44.639017 \\
0.790269 & -0.202302
\end{array}\right]} \\
{\left[\begin{array}{ll}
-0.473153 & -0.662478 \\
-0.001435 & -0.617223
\end{array}\right]} & {\left[\begin{array}{ll}
22.544374 & -12.856893 \\
44.324429 & -23.946844
\end{array}\right]} \\
{\left[\begin{array}{ll}
-0.473153 & -0.662478 \\
-0.001435 & -0.617223
\end{array}\right]} & {\left[\begin{array}{ll}
21.544374 & -12.856893 \\
44.324429 & -24.946844
\end{array}\right]}
\end{array}\right.}
\end{aligned}
$$

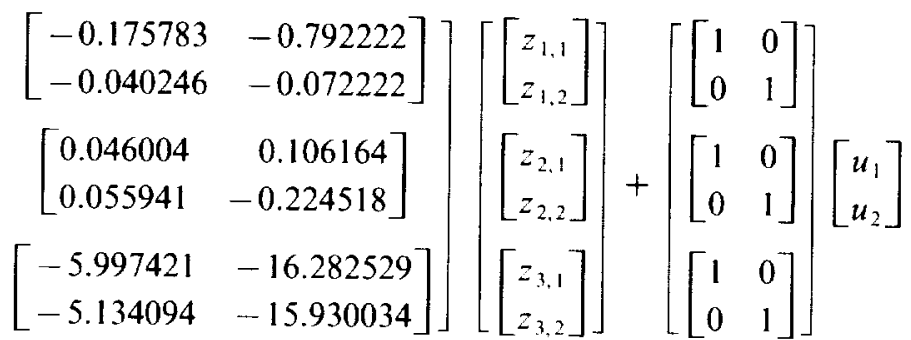




$$
\left[\begin{array}{l}
y_{1} \\
y_{2} \\
y_{3}
\end{array}\right]=\left[\left[\begin{array}{rr}
0.029679 & 0.095386 \\
-0.019033 & 0.224562 \\
0.010361 & 0.196506
\end{array}\right]\left[\begin{array}{rr}
-6.737767 & 3.651197 \\
1.817531 & -1.093386 \\
0.235814 & -0.184328
\end{array}\right]\right.
$$

$$
\left.\left[\begin{array}{ll}
0.009688 & 0.074317 \\
0.005302 & 0.011424 \\
0.022325 & 0.012122
\end{array}\right]\right]\left[\begin{array}{l}
{\left[\begin{array}{l}
z_{1.1} \\
z_{1,2}
\end{array}\right]} \\
{\left[\begin{array}{l}
z_{2.1} \\
z_{2,2}
\end{array}\right]} \\
{\left[\begin{array}{l}
z_{3.1} \\
z_{3,2}
\end{array}\right]}
\end{array}\right]
$$

Using (62) with $\mathbf{A}_{i, j}$ extracted from Table IV, we obtain the block similarity transformation matrix for relating the block state vectors $\mathbf{z}$ and $\mathbf{x}$ in (54) as follows :

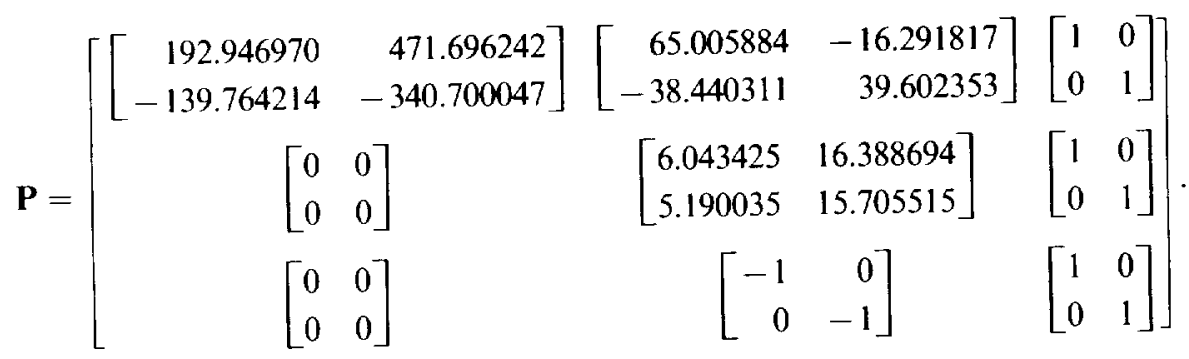

Based on the model reduction method proposed in this paper, the second-degree reduced MFD model, $\hat{\mathbf{G}}_{2}(s)$, derived from the matrix partial quotients, $\mathbf{H}_{i}$, $i=1,2,3,4$ is formed as

$$
\begin{gathered}
\mathbf{G}_{2}(s)=\left[\left[\begin{array}{rr}
-2.396027 & 1.321639 \\
-10.902575 & 5.937033 \\
-7.941521 & 4.336653
\end{array}\right]+\left[\begin{array}{rr}
-6.708088 & 3.746583 \\
1.798498 & -0.868824 \\
0.246175 & 0.012178
\end{array}\right] s\right] \\
\cdot\left[\left[\begin{array}{rr}
-0.837195 & 0.692416 \\
-26.785352 & 14.610068
\end{array}\right]+\left[\begin{array}{ll}
-22.071221 & 13.519371 \\
-44.322994 & 24.564066
\end{array}\right] s+\left[\begin{array}{ll}
1 & 0 \\
0 & 1
\end{array}\right] s^{2}\right]^{-1} \\
=\left[\begin{array}{lll}
\hat{g}_{1,1}(s) & \hat{g}_{1,2}(s) & \hat{g}_{1,3}(s) \\
\hat{g}_{2,1}(s) & \hat{g}_{2,2}(s) & \hat{g}_{2,3}(s)
\end{array}\right]
\end{gathered}
$$

where

$$
\begin{aligned}
& \hat{g}_{1,1}(s)=\left(0.394446+2.070750 s-1.114170 s^{2}-6.708088 s^{3}\right) / \hat{d}(s) \\
& \hat{g}_{1.2}(s)=\left(0.552578+4.730760 s+9.319107 s^{2}+3.746583 s^{3}\right) / \hat{d}(s) \\
& \hat{g}_{2,1}(s)=\left(-0.261851-1.660099 s-5.233055 s^{2}+1.798498 s^{3}\right) / \hat{d}(s)
\end{aligned}
$$


Tong-Yi Guo et al.

$$
\begin{aligned}
\hat{g}_{2,2}(s) & =\left(2.578663+15.840457 s+0.798490 s^{2}-0.868824 s^{3}\right) / \hat{d}(s) \\
\hat{g}_{3,1}(s) & =\left(0.132620+1.060232 s-1.354695 s^{2}+0.246175 s^{3}\right) / \hat{d}(s) \\
\hat{g}_{3,2}(s) & =\left(1.868212+11.468484 s+0.739738 s^{2}+0.012178 s^{3}\right) / \hat{d}(s) \\
\hat{d}(s) & =6.315131+49.784100 s+70.832926 s^{2}+2.492846 s^{3}+s^{4} .
\end{aligned}
$$

Furthermore, the multipoint Cauer MCFE state-space model corresponding to $\hat{\mathbf{G}}_{2}(s)$ can be readily obtained as

$$
\begin{aligned}
& {\left[\begin{array}{l}
{\left[\begin{array}{l}
\dot{\hat{z}}_{1,1} \\
\dot{\hat{z}}_{1,2}
\end{array}\right]} \\
{\left[\begin{array}{l}
\dot{\hat{z}}_{2,1} \\
\dot{z}_{2,2}
\end{array}\right]}
\end{array}\right]=\left[\begin{array}{ll}
{\left[\begin{array}{ll}
-0.473153 & -0.662478 \\
-0.001435 & -0.617223
\end{array}\right]} & {\left[\begin{array}{rr}
81.728620 & -44.639017 \\
0.790269 & -0.202302
\end{array}\right]} \\
{\left[\begin{array}{ll}
-0.473153 & -0.662478 \\
-0.001435 & -0.617223
\end{array}\right]} & {\left[\begin{array}{lll}
22.544374 & -12.856893 \\
44.324429 & -23.946844
\end{array}\right]}
\end{array}\right]} \\
& \cdot\left[\begin{array}{l}
{\left[\begin{array}{l}
\hat{z}_{1,1} \\
\hat{z}_{1,2}
\end{array}\right]} \\
{\left[\begin{array}{l}
\hat{z}_{2,1} \\
\hat{z}_{2,2}
\end{array}\right]}
\end{array}\right]+\left[\begin{array}{ll}
{\left[\begin{array}{ll}
1 & 0 \\
0 & 1
\end{array}\right]} \\
{\left[\begin{array}{ll}
1 & 0 \\
0 & 1
\end{array}\right]}
\end{array}\right]\left[\begin{array}{l}
u_{1} \\
u_{2}
\end{array}\right]
\end{aligned}
$$

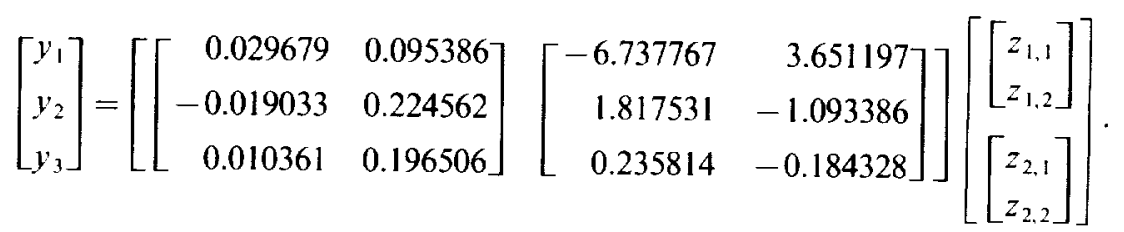

The Taylor series expansions of $\hat{\mathbf{G}}_{2}(s)$ around $s=0,1,2$ are, respectively, evaluated as

$$
\begin{aligned}
\hat{\mathbf{G}}_{2}(s)= & {\left[\begin{array}{rr}
0062460 & 0.087501 \\
-0.041464 & 0.408331 \\
0021000 & 0.295831
\end{array}\right]+\left[\begin{array}{rr}
-0.164492 & 0.059321 \\
0.063997 & -0.710663 \\
0.002335 & -0.516094
\end{array}\right] s } \\
& +\left[\begin{array}{rr}
0.419732 & 0.026594 \\
-0.868085 & 1.148819 \\
-0.468475 & 0.867507
\end{array}\right] s^{2}+\cdots \\
= & {\left[\begin{array}{rr}
-0.041074 & 0.140686 \\
-0.041070 & 0.140685 \\
0.000647 & 0.108021
\end{array}\right]+\left[\begin{array}{rr}
-0.091599 & 0.046459 \\
0.012294 & -0.105179 \\
-0.007988 & -0.068514
\end{array}\right](s-1) } \\
& +\left[\begin{array}{rr}
0.006230 & -0.005601 \\
0.008666 & 0.058842 \\
0.007286 & 0.042725
\end{array}\right](s-1)^{2}+\cdots
\end{aligned}
$$




$$
\begin{aligned}
= & {\left[\begin{array}{rr}
-0.126037 & 0.181728 \\
-0.023818 & 0.071745 \\
-0.002814 & 0.065532
\end{array}\right]+\left[\begin{array}{rr}
-0.077841 & 0.035700 \\
0.019754 & -0.046413 \\
-0.000689 & -0.026610
\end{array}\right](s-2) } \\
& +\left[\begin{array}{rr}
0.007584 & -0.005306 \\
0.000869 & 0.014214 \\
0.001655 & 0.009714
\end{array}\right](s-2)^{2}+\cdots
\end{aligned}
$$

Comparing the above series with those of $\mathbf{G}(s)$, we can verify that the partial matrix Padé properties of $\hat{\mathbf{G}}(s)$ given in (46a) and (47a) match those of $\mathbf{G}(s)$.

For comparison of the time responses between reduced-degree models and $\mathbf{G}(s)$, the second-degree reduced MFD obtained by the single-point Cauer second form of the MCFE (12) is given below:

$$
\begin{aligned}
& \hat{\mathbf{G}}_{2}^{*}(s)=\left[\left[\begin{array}{rr}
-2.446550 & 1.375684 \\
-11.180131 & 6.225057 \\
-8.136202 & 4.539832
\end{array}\right]+\left[\begin{array}{rr}
-6.893735 & 3.936407 \\
1.825865 & -0.899738 \\
0.213142 & 0.045311
\end{array}\right] s\right] \\
& \cdot\left[\left[\begin{array}{rr}
-0.711690 & 0.584829 \\
-27.452343 & 15.304513
\end{array}\right]+\left[\begin{array}{ll}
-22.590895 & 14.079862 \\
-45.510647 & 25.793547
\end{array}\right] s\right. \\
& \left.+\left[\begin{array}{ll}
1 & 0 \\
0 & 1
\end{array}\right] s^{2}\right]^{\prime}=\left[\begin{array}{lll}
\hat{g}_{1,1}^{*}(s) & \hat{g}_{1,2}^{*}(s) & \hat{g}_{1,3}^{*}(s) \\
\hat{g}_{2,1}^{*}(s) & \hat{g}_{2,2}^{*}(s) & \hat{g}_{2,3}^{*}(s)
\end{array}\right]
\end{aligned}
$$

where

$$
\begin{aligned}
\hat{g}_{1,1}^{*}(s) & =\left(0.322474+2.061388 s-1.111976 s^{2}-6.893375 s^{3}\right) / \hat{d}^{*}(s) \\
\hat{g}_{1,2}^{*}(s) & =\left(0.451753+4.599323 s+9.511552 s^{2}+3.936407 s^{3}\right) / \hat{d}^{*}(s) \\
\hat{g}_{2,1}^{*}(s) & =\left(-0.214073-1.824823 s-5.032249 s^{2}+1.825865 s^{3}\right) / \hat{d}^{*}(s) \\
\hat{g}_{2,2}^{*}(s) & =\left(2.108154+16.357619 s+0.843013 s^{2}-0.899738 s^{3}\right) / \hat{d}^{*}(s) \\
\hat{g}_{3,1}^{*}(s) & =\left(0.108422+1.255139 s-0.576348 s^{2}+0.213142 s^{3}\right) / \hat{d}(s) \\
\hat{g}_{3,2}^{*}(s) & =\left(1.527333+11.840828 s+0.515189 s^{2}+0.045311 s^{3}\right) / \hat{d}^{*}(s) \\
\hat{d}^{*}(s) & =5.162856+49.041488 s+72.677136 s^{2}+3.202651 s^{3}+s^{4} .
\end{aligned}
$$

The values of the impulse response energy (18) for transfer function elements and outputs of the original system and those of the reduced-degree MFD are listed in Table VI. The values of the integral squared-error of the impulse and the unit-step time responses for each output of the reduced models are shown in Table VII. The above values are usually taken as criteria for choosing proper reduced-degree models. The unit-step time responses for each output of the original system $\mathbf{G}(s)$ and the reduced-degree models $\hat{\mathbf{G}}_{2}(s)$ are plotted in Fig. 3 . It is observed that the time-responses of the reduced model are close to those of the original system. 
Tong-Yi Guo et al.

TABLE VI

The values of impulse response energy of the original and reduceddegree models

\begin{tabular}{ccrr} 
& $\hat{\mathbf{G}}_{2}^{*}(s)$ & $\hat{\mathbf{G}}_{2}(s)$ & \multicolumn{1}{c}{$\mathbf{G}(s)$} \\
\hline$g_{1,1}(s)$ & 9.493938 & 12.707962 & 14.771495 \\
$g_{2.1}(s)$ & 0.748100 & 1.037583 & 1.283513 \\
$g_{3.1}(s)$ & 0.008769 & 0.022492 & 0.042638 \\
$g_{1.2}(s)$ & 3.219912 & 4.134660 & 4.683292 \\
$g_{2.2}(s)$ & 0.298879 & 0.382659 & 0.448826 \\
$g_{3.2}(s)$ & 0.027614 & 0.032275 & 0.037869 \\
$y_{1}(s)$ & 2.039829 & 2.888713 & 3.421429 \\
$y_{2}(s)$ & 0.191937 & 0.267885 & 0.331350 \\
$y_{3}(s)$ & 0.030720 & 0.032574 & 0.038061 \\
\hline
\end{tabular}

TABLE VII

The integral squared-errors of the impulse and unit-step responses between the original and reduced-degree models

\begin{tabular}{ccccccc}
\hline & \multicolumn{3}{c}{ Impulse response } & \multicolumn{3}{c}{ Unit-step response } \\
& $y_{1}(s)$ & $y_{2}(s)$ & $y_{3}(s)$ & $y_{1}(s)$ & $y_{2}(s)$ & $y_{3}(s)$ \\
\hline$\hat{\mathbf{G}}_{2}^{*}(s)$ & 0.467087 & 0.045071 & 0.003970 & 0.006885 & 0.000700 & 0.000136 \\
$\hat{\mathbf{G}}_{2}(s)$ & 0.135860 & 0.014271 & 0.001245 & 0.002030 & 0.000257 & 0.000098 \\
\hline
\end{tabular}

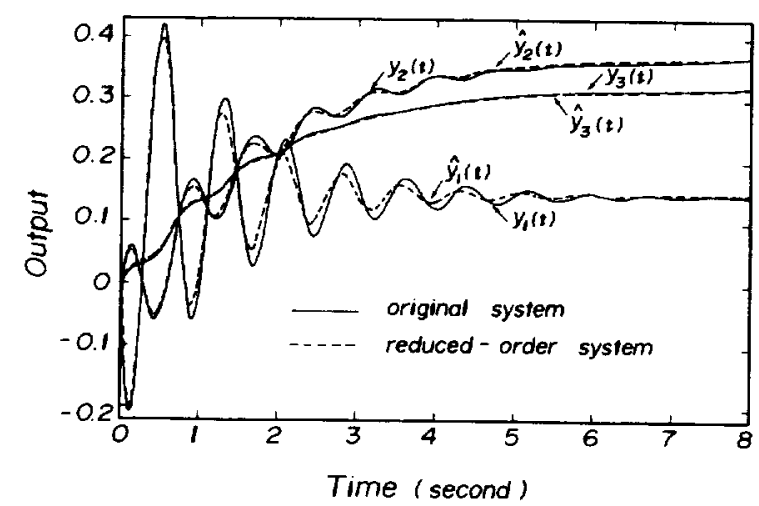

FIG. 3. Unit-step responses of the original and reduced-degree models. 


\section{Conclusions}

A matrix continued-fraction methodology has been established for modeling and model reduction of multivariable systems having an unequal number of inputs and outputs. The developed methodology is based on the multipoint Cauer matrix continued-fraction expansion and the matrix pseudo-inverse, and is applicable to obtain both the frequency-domain reduced-degree MFDs and time-domain reduced-dimensional state-space models. The connection of the presented multipoint Cauer MCFE method involving matrix pseudo-inverse to the multipoint matrix Padé approximation method is also established. Although the algorithms in the paper are derived for a right MFD, the extension to a left MFD is straightforward.

The use of a multipoint continued fraction expansion gives good reduced-order models that provide satisfactory approximation of the original system not only in the transient response but also in the steady-state response. Although the stability of the reduced models by the multipoint Cauer MCFE is not completely guaranteed, the flexibility of choosing expansion points provides the highest probability for finding stable reduced-order models (19).

\section{References}

(1) U. M. Al-Saggaf and G. F. Franklin, "Model reduction via balanced realizations: an extension and frequency weighting techniques", IEEE Trans. Autom. Control, Vol. AC-33, pp. 687-692, 1988.

(2) C. F. Chen, "Model reduction of multivariable control systems by means of matrix continued fractions". Int. J. Control, Vol. 20, pp. 225-238, 1974.

(3) B. C. Moore, "Model reduction for multivariable systems", Int. J. Control, Vol. 20, pp. 57-64, 1974.

(4) B. C. Moore, "Principal component analysis in linear systems : controllability, observability, and model reduction", IEEE Trans. Autom. Control, Vol. AC-26, pp. $17-32,1981$.

(5) R. J. Ober, "Balanced realizations: canonical form, parameterization, model reduction", Int. J. Control, Vol. 46, pp. 643-670, 1987.

(6) M. G. Safonov and R. Y. Chiang, "Model reduction for robust control: a Schur relative crror method", Int. J. Adapt. Signal Process., Vol. 2, pp. 259-272, 1988.

(7) M. Y. Chen and C. Hwang, "Multipoint matrix continued-fraction description for linear multivariable system reduction", Proc. Natl. Sci. Counc. ROC(A), Vol. 12, pp. $117-127,1988$

(8) M. J. Goldman, W. J. Porras and C. T. Lenones, "Multivariable systems reduction via Cauer forms", Int. J. Control, Vol. 34, pp. 623-650, 1981.

(9) L. S. Shieh and F. F. Gaudiano, "Matrix continued fraction expansion and inversion by the generalized matrix Routh algorithm", Int. J. Control, Vol. 20, pp. 727-737, 1974.

(10) L. S. Shieh and F. F. Gaudiano, "Some properties and applications of matrix continued fraction", IEEE Trans. Circuits Syst., Vol. CAS-22, pp. 721-728, 1975.

(11) L. S. Shieh, Y. J. Wei, R. E. Yates and J. P. Leonard, "Two methods for simplifying multivariable systems with various numbers of inputs and outputs", Int. $J$. Systems Sci., Vol. 7, pp. 501-512, 1976.

(12) L. S. Shieh, C. G. Patel and H. Z. Chow, "Additional properties and application of matrix continued fraction", Int. J. Systems Sci., Vol. 8, pp. 97-109, 1977. 
Tong-Yi Guo et al.

(13) L. S. Shieh, F. R. Chang and R. E. Yates, "The generalized matrix continued-fraction descriptions and their applications to model simplification", Int. J. Systems Sci., Vol. 17, pp. 1-19, 1986.

(14) C. Hwang and M. Y. Chen, "A multipoint continued-fraction expansion for linear system reduction", IEEE Trans. Autom. Control, Vol. AC-31, pp. 648-651, 1986.

(15) R. V. Patel, "Computation of matrix fraction descriptions of linear time-invariant systems, IEEE Trans. Autom. Control, Vol. AC-26, pp. 148-161, 1981.

(16) P. Lancaster, "Theory of Matrices", Academic Press, New York, 1969.

(17) L. S. Shieh and T. Y. Tsay, "Transformation of a class of multivariable control systems to block companion forms", IEEE Trans. Autom. Control, Vol. AC-27, pp. 199203, 1982.

(18) M. F. Hutton and B. Friedland, "Routh approximations for reduced order of linear time-invariant systems", IEEE Trans. Autom. Control, Vol. AC-20, pp. 329-337, 1975.

(19) T. N. Lucas, "New results on relationships between multipoint Padé approximation and stability preserving methods in model reduction", Int. J. Control, Vol. 20, pp. 1267-1274, 1989.

Received : 30 August 1993

Accepted: 11 October 1993 